\title{
A SPLICING FORMULA FOR THE LMO INVARIANT
}

\author{
GWÉNAËL MASSUYEAU AND DELPHINE MOUSSARD
}

\begin{abstract}
We prove a "splicing formula" for the LMO invariant, which is the universal finite-type invariant of rational homology 3-spheres. Specifically, if a rational homology 3sphere $M$ is obtained by gluing the exteriors of two framed knots $K_{1} \subset M_{1}$ and $K_{2} \subset M_{2}$ in rational homology 3-spheres, our formula expresses the LMO invariant of $M$ in terms of the Kontsevich-LMO invariants of $\left(M_{1}, K_{1}\right)$ and $\left(M_{2}, K_{2}\right)$. The proof uses the techniques that Bar-Natan and Lawrence developed to obtain a rational surgery formula for the LMO invariant. In low degrees, we recover Fujita's formula for the Casson-Walker invariant and we observe that the second term of the Ohtsuki series is not additive under "standard" splicing. The splicing formula also works when each $M_{i}$ comes with a link $L_{i}$ in addition to the knot $K_{i}$, hence we get a "satellite formula" for the Kontsevich-LMO invariant.
\end{abstract}

\section{Contents}

1. Introduction

2. Splicing and surgery

3. Splicing formula

4. Low degree formulas

5. Splicing and satellite operations

6. The case of knots that are not trivial in homology

Appendix A. Signatures of tridiagonal matrices

References

\section{INTRODUCTION}

The LMO invariant of closed oriented 3-manifolds was constructed by Le, Murakami and Ohtsuki [LMO98]: from the Kontsevich integral of a surgery link, they derived a quantity which is invariant under Kirby moves. According to Le [Le97], the LMO invariant of integral homology 3-spheres is universal among all $\mathbb{Q}$-valued finite-type invariants in the sense of [096a, GGP01, H00]. A more general result is true for rational homology 3-spheres whose first homology groups have a fixed cardinality [M12b, M14]. In the case of a rational homology 3-sphere $M$, the LMO invariant of $M$ coincides with its Aarhus integral $Z(M)$ [BGRT02, BGRT04]. The invariant $Z(M)$ takes values in a graded vector space of trivalent diagrams, which is denoted by $\mathcal{A}(\emptyset)$. Its first non-trivial term, the coefficient of the $\theta$-shaped diagram, is given by the Casson-Walker invariant $\lambda_{\mathrm{W}}(M)$ as normalized in [W92]:

$$
Z(M)=\emptyset+\frac{\lambda_{\mathrm{W}}(M)}{4} \ominus+\left(\begin{array}{c}
\text { trivalent diagrams } \\
\text { with } \geq 4 \text { vertices }
\end{array}\right) \in \mathcal{A}(\emptyset) .
$$

This research has been funded by the project "ITIQ-3D" of the Région Bourgogne Franche-Comté. G.M. is partly supported by the project "AlMaRe" (ANR-19-CE40-0001-01) and by the EIPHI Graduate School (ANR-17-EURE-0002). 
The splicing operation is the general procedure by which, given two framed knots in oriented 3-manifolds, one creates a new 3-manifold by gluing the exteriors of these knots. The term "splicing" is sometimes used in the literature for what is called below the standard splicing, namely the case when the gluing homeomorphism identifies the meridian of each of the two knots with the parallel of the other one. Standard splicing has the property to preserve the class of integral homology 3-spheres (a knot in such a manifold being framed with its "preferred" parallel). Another special case of splicing is given by the rational surgery on a knot.

The main result of this paper expresses the LMO invariant of a rational homology 3-sphere that is defined as the splice of two framed knots in rational homology 3 -spheres, in terms of the Kontsevich-LMO invariants of the two knots. For simplicity, we will first consider the case of null-homologous knots (which are framed with the "preferred" parallel).

Theorem. Let $K_{1} \subset M_{1}$ and $K_{2} \subset M_{2}$ be null-homologous knots in rational homology 3spheres. Consider a splice $M$ of $\left(M_{1}, K_{1}\right)$ and $\left(M_{2}, K_{2}\right)$ that is also a rational homology 3-sphere. Then, we have

$$
Z(M)=\omega \exp \left(\frac{1}{48}\left(-S\left(\frac{p}{r}\right)+\frac{p+s}{r}\right) \ominus\right)\left\langle\partial_{p, r}\left(Z^{\not \alpha}\left(K_{1}\right)\right), \partial_{s, r}\left(Z^{\not \alpha}\left(K_{2}\right)\right)\right\rangle_{r}
$$

where $Z^{\text {W }}\left(K_{i}\right)$ is the "wheeled" version of the Kontsevich-LMO invariant of $K_{i} \subset M_{i}$, the integers $p, r, s$ depend on the gluing homeomorphism, the term $\omega \in \mathcal{A}(\emptyset)$ is a constant, $S(p / r)$ is a Dedekind symbol and $\partial_{p, r}, \partial_{s, r},\langle-,-\rangle_{r}$ are diagrammatic operations depending only on the indicated integers $p, r, s$.

The reader is referred to Theorem 3.1 for a precise statement of this "splicing formula". Although the LMO invariant does not separate rational homology 3-spheres [BL04], it is still unknown whether it separates integral homology 3-spheres; nevertheless, Bar-Natan and Lawrence proved that it does separate Seifert fibered spaces that are integral homology $3-$ spheres. They also computed the LMO invariant of all lens spaces. We expect that the splicing formula will be useful to compute (to some extent) the LMO invariant of new families of rational homology 3-spheres. We particularly think of graph manifolds (within this homology type) since they are obtained from Seifert fibered spaces by repeated splicings along their fibers. A possible outcome of this study would be to decide whether the LMO invariant also distinguishes graph manifolds that are integral homology 3-spheres.

The paper is organized as follows. In Section 2, we describe the splice of two framed knots in closed oriented 3-manifolds - say $K_{1} \subset M_{1}$ and $K_{2} \subset M_{2}$ - as the result of the surgery along a framed link $K_{1} \sqcup H \sqcup K_{2}$ in the connected sum $M_{1} \sharp M_{2}$, where $H$ is a "chain" of Hopf links "clasping" $K_{1}$ and $K_{2}$. This surgery description of the splicing operation is wellknown in, at least, two special cases: for standard splicings and for rational surgeries. Even in its most general form, the reader could deduce it from [G75, §5.2] by determining the handle decomposition of the plumbed 4-manifold that is constructed there. Here the surgery description of a splice is proved by purely 3-dimensional arguments.

In Section 3, we prove the above theorem. Starting with the surgery description of a splice, we use the techniques that Bar-Natan and Lawrence developed to produce a rational surgery formula for the LMO invariant [BL04]; their work relies itself on the "Wheels and Wheeling" conjectures proved in [BLT03]. The rational surgery formula is reproved here as a consequence of the splicing formula (Corollary 3.3).

In Section 4, the splicing formula is made explicit in low degrees. In degree two, we recover Fujita's splicing formula [F93] for the Casson-Walker invariant $\lambda_{\mathrm{W}}$, which involves the second 
derivatives of the Alexander polynomial of knots (Proposition 4.1). In degree four, we obtain a splicing formula for the second term $\lambda_{2}$ of the Ohtsuki series which, in addition to the second and fourth derivatives of the Alexander polynomial, needs the third coefficient of the expansion of the Jones polynomial (Proposition 4.4). It turns out that, in contrast with $\lambda_{\mathrm{W}}$, the invariant $\lambda_{2}$ is not additive with respect to standard splicing.

The last two sections provide more general versions of the splicing formula, which will be needed for the above-mentioned project of studying the LMO invariant of graph manifolds. In Section 5, we consider the situation where each $M_{i}$ in the above theorem comes with an additional link $L_{i}$ (disjoint from $K_{i}$ ). Then, the Kontsevich-LMO invariant of $L_{1} \sqcup L_{2} \subset M$ is expressed by the same formula ( 1.1) in terms of the Kontsevich-LMO invariants of $K_{1} \sqcup L_{1} \subset$ $M_{1}$ and $K_{2} \sqcup L_{2} \subset M_{2}$ (Theorem 5.1). As explained in the monograph [EN85], splicing is a fundamental operation in knot theory: indeed, splicing subsumes all satellite operations (such as connected sum, cabling, Whitehead doubling, etc). Thus, we give a "satellite formula" for the Kontsevich-LMO invariant (Corollary 5.3) and we derive from this a result of Suetsugu [S96]. Finally, we prove in Section 6 a generalization of the above theorem where each knot $K_{i}$ is allowed to be non-trivial in homology. Then formula (1.1) extends by also taking into account the self-linking numbers of $K_{1}$ and $K_{2}$ (Theorem 6.2).

The paper ends with an appendix which collects useful facts about tridiagonal matrices and their signatures in terms of Dedekind sums.

Conventions. Throughout the paper, all manifolds are assumed to be compact, connected and oriented. The boundary of a manifold (if any) is oriented with the "outward normal first" convention.

Unless otherwise stated, all knots are oriented. Given a framed knot $K$ in a $3-$ manifold $M$, the parallel $\rho(K)$ of $K$ (defining the framing) inherits from $K$ an orientation and the meridian $\mu(K)$ of $K$ is oriented so that $\operatorname{lk}(K, \mu(K))=+1$. Given a framed $\operatorname{link} L$ in a 3 -manifold $M$, the manifold obtained from $M$ by surgery on $L$ is denoted $M_{L}$.

\section{SPLICING AND SURGERY}

Let $M_{1}$ and $M_{2}$ be closed 3-manifolds, and let $K_{1} \subset M_{1}$ and $K_{2} \subset M_{2}$ be knots. For $i=1,2$, let $\mathrm{N}\left(K_{i}\right)$ be a tubular neighborhood of $K_{i}$ and set $X_{i}:=M_{i} \backslash \operatorname{Int}\left(\mathrm{N}\left(K_{i}\right)\right)$. Given an orientation-reversing homeomorphism

$$
f: \partial \mathrm{N}\left(K_{1}\right) \stackrel{\cong}{\longrightarrow} \partial \mathrm{N}\left(K_{2}\right)
$$

define the splice of $\left(M_{1}, K_{1}\right)$ and $\left(M_{2}, K_{2}\right)$ along $f$ as the closed 3-manifold

$$
M:=X_{1} \bigcup_{f} X_{2} \text {. }
$$

The goal of this section is to describe $M$ as a surgery along a framed link in the connected sum $M_{1} \sharp M_{2}$.

Remark 2.1. There is a more general "self-splicing" operation, which is defined as follows: given a closed (possibly disconnected) 3-manifold $M_{0}$, two disjoint knots $K_{1}, K_{2} \subset M_{0}$, and an orientation-reversing homeomorphism $f: \partial \mathrm{N}\left(K_{1}\right) \rightarrow \partial \mathrm{N}\left(K_{2}\right)$, the self-splice $M$ of $\left(M_{0}, K_{1}, K_{2}\right)$ is the result of self-gluing $M_{0} \backslash \operatorname{Int}\left(\mathrm{N}\left(K_{1}\right) \cup \mathrm{N}\left(K_{2}\right)\right)$ using $f$. Let $M_{i}$ be the connected component of $M_{0}$ containing $K_{i}$ for $i=1,2$, and assume for simplicity that $M_{0}=M_{1} \cup M_{2}$. If $M_{1} \neq M_{2}$, then $M$ is the splice of $\left(M_{1}, K_{1}\right)$ and $\left(M_{2}, K_{2}\right)$. If $M_{1}=M_{2}$, 
then $M$ is not a rational homology 3 -sphere even if $M_{0}$ is assumed to be so. Since we are only interested in rational homology 3-spheres in this paper, we will not further consider self-splices.

Fix a "model" $\mathbb{T}$ of the 2 -dimensional torus, together with a basis $(\alpha, \beta)$ of its fundamental group. The surface $\mathbb{T}$ is oriented so that the intersection number $\alpha \cdot \beta$ is +1 . If some identifications $\mathbb{T} \cong-\partial \mathrm{N}\left(K_{1}\right)$ and $\mathbb{T} \cong \partial \mathrm{N}\left(K_{2}\right)$ are fixed, then the splice ( 2.1) can be achieved by gluing the mapping cylinder of an orientation-preserving self-homeomorphism of $\mathbb{T}$ to $X_{1} \sqcup X_{2}$. We will use this point of view to get a surgery description of the splicing operation.

To this aim, we study toroidal cobordisms, namely 3 -manifolds $C$ whose boundary $\partial C=$ $\left(-\partial_{-} C\right) \sqcup \partial_{+} C$ is decomposed into two parts, $\partial_{+} C$ and $\partial_{-} C$, each being identified to $\mathbb{T}$. The composition $D \circ C$ of two toroidal cobordisms, $C$ and $D$, is defined by gluing $C$ to $D$ using the given identifications $\partial_{+} C \cong \mathbb{T} \cong \partial_{-} D$. There is a one-to-one correspondence between pairs $(M, J)$, where $J$ is a 2 -component framed link in a closed 3 -manifold $M$, and toroidal cobordisms $C$. Specifically, the cobordism corresponding to a 2-component framed link $J=J^{\prime} \sqcup J^{\prime \prime}$ in a closed 3-manifold $M$ is its exterior

$$
C:=M \backslash\left(\operatorname{Int}\left(\mathrm{N}\left(J^{\prime}\right)\right) \sqcup \operatorname{Int}\left(\mathrm{N}\left(J^{\prime \prime}\right)\right)\right)
$$

where $\partial_{-} C:=\partial \mathrm{N}\left(J^{\prime}\right)$ is identified to $\mathbb{T}$ by $\left(\mu\left(J^{\prime}\right), \rho\left(J^{\prime}\right)\right) \mapsto(\alpha, \beta)$ and $\partial_{+} C:=-\partial \mathrm{N}\left(J^{\prime \prime}\right)$ is identified to $\mathbb{T}$ by $\left(\mu\left(J^{\prime \prime}\right), \rho\left(J^{\prime \prime}\right)\right) \mapsto(\alpha,-\beta)$. Here we use the conventions that have been set at the very end of the introduction.

In this way, toroidal cobordisms can be presented by a surgery link in the complement of the trivial 2-component framed link in $S^{3}$. For instance, Figure 1 represents $M$ as the 3 -manifold obtained by surgery on the black framed unoriented link, while $J^{\prime}$ and $J^{\prime \prime}$ are the copies in $M$ of the blue ( $\star$-marked) knot and the red $(\bullet-$ marked) knot, respectively; each $k \in \mathbb{Z}$ decorating a black (unmarked) component is here to mean that we are performing a surgery with a framing number that differs by $k$ from the "blackboard framing". In the sequel, we shall use these diagrammatic conventions for surgery presentations.

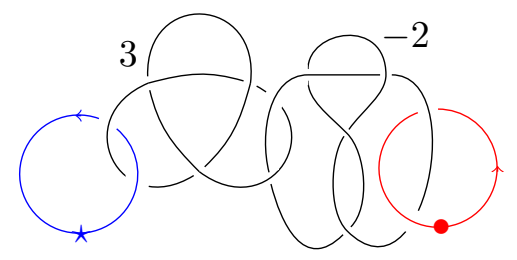

FIgURE 1. Surgery presentation of a toroidal cobordism

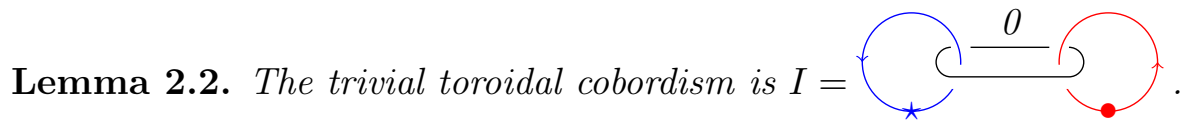

Proof. The manifold obtained by surgery is $S^{2} \times S^{1}$; the knots $J^{\prime}$ and $J^{\prime \prime}$ that correspond to the blue $(\star-$ marked $)$ component and to the red $(\bullet-$ marked $)$ component, respectively, are of the form $\{*\} \times S^{1}$. Hence the cobordism is an annulus times $S^{1}$, namely a thickened torus. Moreover, the meridian $\mu\left(J^{\prime}\right)$ can be slid over the disk glued by surgery to get $\mu\left(J^{\prime \prime}\right)$. Finally, $\rho\left(J^{\prime}\right)$ is clearly homotopic to $-\rho\left(J^{\prime \prime}\right)$. 
Lemma 2.3. Let $C$ and $D$ be toroidal cobordisms, which are respectively associated to pairs $(M, J)$ and $(N, L)$ of framed links in 3-manifolds. Then the composed cobordism $D \circ C$ is associated to the pair $\left((M \sharp N)_{J^{\prime \prime} \sharp L^{\prime}}, J^{\prime} \sqcup L^{\prime \prime}\right)$.

Proof. Perform the connected-sum inside the tubular neighborhoods $\mathrm{N}\left(J^{\prime \prime}\right)$ and $\mathrm{N}\left(L^{\prime}\right)$. Then the cobordism associated to $\left((M \sharp N)_{J^{\prime \prime} \sharp L^{\prime}}, J^{\prime} \sqcup L^{\prime \prime}\right)$ is

$$
C^{\prime} \circ\left(\mathrm{N}\left(J^{\prime \prime}\right) \sharp \mathrm{N}\left(L^{\prime}\right)\right)_{J^{\prime \prime \sharp L^{\prime}}} \circ C .
$$

It is easily checked that $\left(\mathrm{N}\left(J^{\prime \prime}\right) \sharp \mathrm{N}\left(L^{\prime}\right)\right)_{J^{\prime \prime} \sharp L^{\prime}}$ is the trivial cobordism (e.g., using Lemma 2.2).

For any $a \in \mathbb{Z}$, we introduce the following toroidal cobordisms:

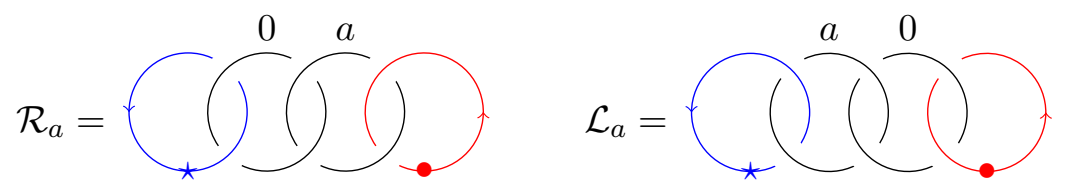

Let us compute the composition $\mathcal{L}_{-a} \circ \mathcal{R}_{a}$ using Lemma 2.3:

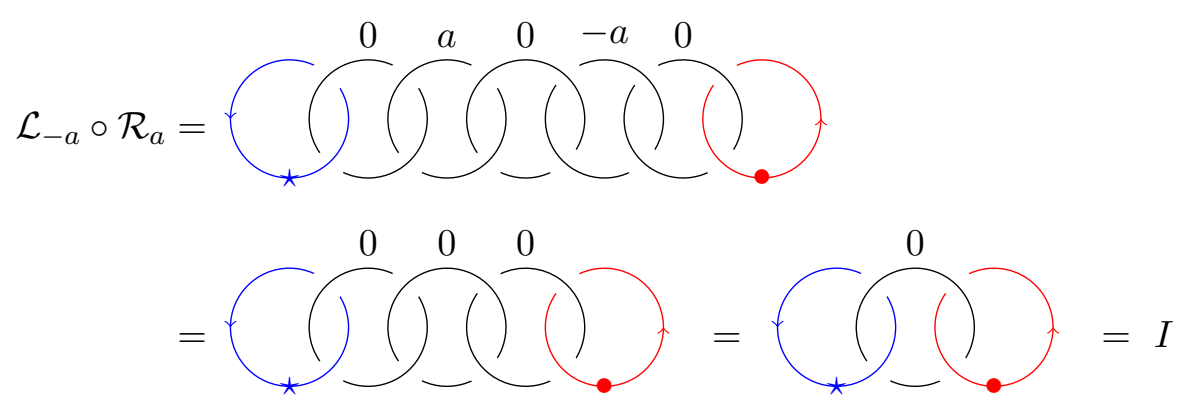

The second and third equalities are given by slam-dunk moves as represented in the right hand side of Figure 2 (see [CG88] for the left hand side and deduce the right hand side by sliding first the rightmost component on the leftmost one). We deduce that the cobordisms $\mathcal{R}_{a}$ and $\mathcal{L}_{a}$ are invertible, which implies that they are mapping cylinders. We focus on the $\mathcal{L}_{a}$ and compute their action in homology.
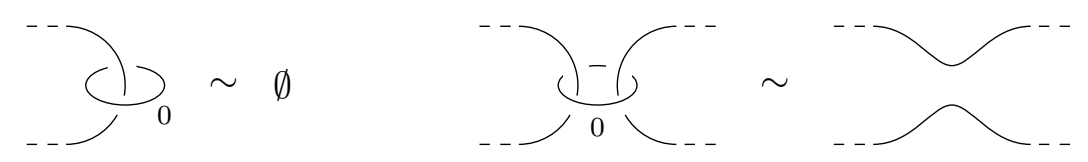

Figure 2. A slam-dunk move and a corollary

Lemma 2.4. The action of the cobordism $\mathcal{L}_{a}$ on the homology of $\mathbb{T}$ is given by the matrix $\left(\begin{array}{cc}a & -1 \\ 1 & 0\end{array}\right)$ in the basis $(\alpha, \beta)$ of $H_{1}(\mathbb{T} ; \mathbb{Z})$.

Proof. Denote by $L_{a}$ and $L_{0}$ the surgery components of $\mathcal{L}_{a}$ in (2.2) framed by $a$ and 0 respectively, and orient each of them in the counter-clockwise direction. We have the following identities in $H_{1}\left(\mathcal{L}_{a} ; \mathbb{Z}\right)$ :

$$
\rho\left(J^{\prime}\right)=\mu\left(L_{a}\right) \quad \rho\left(L_{a}\right)=\mu\left(J^{\prime}\right)+\mu\left(L_{0}\right) \quad \rho\left(L_{a}\right)+a \mu\left(L_{a}\right)=0
$$




$$
\rho\left(J^{\prime \prime}\right)=\mu\left(L_{0}\right) \quad \rho\left(L_{0}\right)=\mu\left(L_{a}\right)+\mu\left(J^{\prime \prime}\right) \quad \rho\left(L_{0}\right)=0
$$

This gives $\mu\left(J^{\prime}\right)=a \mu\left(J^{\prime \prime}\right)-\rho\left(J^{\prime \prime}\right)$ and $\rho\left(J^{\prime}\right)=-\mu\left(J^{\prime \prime}\right)$, and we can conclude.

Lemma 2.5. For any integers $a_{1}, \ldots, a_{n}$, we have

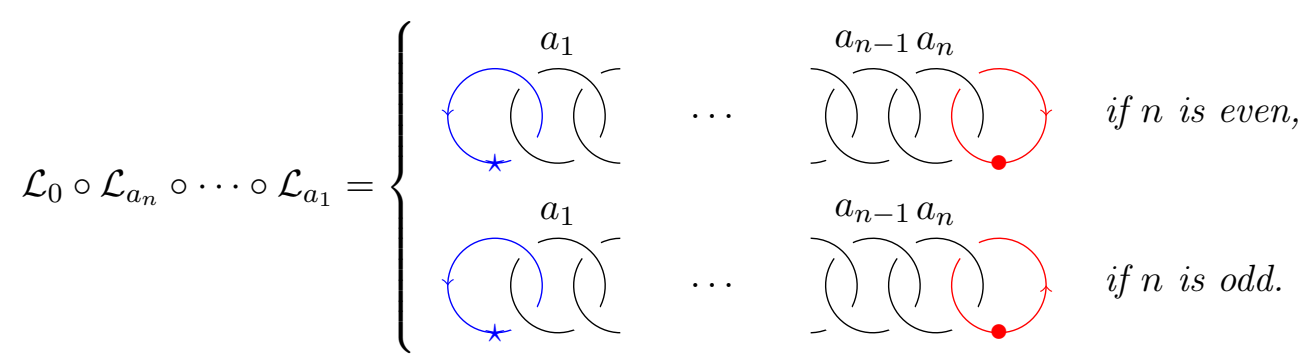

Proof. For any integers $a$ and $b$, we have

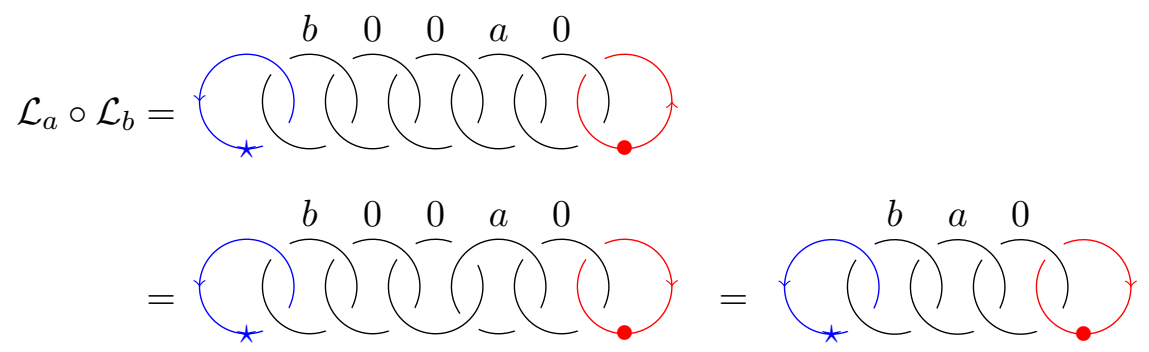

where the first equality is given by Lemma 2.3, the second one by an isotopy and the third one by slam-dunk. For any integers $a_{1}, \ldots, a_{n}, a_{n+1}$, a similar computation gives by induction:

$$
\mathcal{L}_{a_{n+1}} \circ \mathcal{L}_{a_{n}} \circ \cdots \circ \mathcal{L}_{a_{1}}= \begin{cases}a_{1} \\ a_{1}\end{cases}
$$

Setting $a_{n+1}:=0$ and applying a slam-dunk move leads to the result.

We now come back to the splice $M$ of $\left(M_{1}, K_{1}\right)$ and $\left(M_{2}, K_{2}\right)$ defined by an orientationreversing homeomorphism $f: \partial \mathrm{N}\left(K_{1}\right) \rightarrow \partial \mathrm{N}\left(K_{2}\right)$ and we assume that $K_{1}, K_{2}$ are framed. Using the framing, we make the following identifications:

$$
\begin{array}{ll}
-\partial X_{2}=\partial \mathrm{N}\left(K_{2}\right) \stackrel{\cong}{\cong}, & \left(\mu\left(K_{2}\right), \rho\left(K_{2}\right)\right) \longmapsto(\alpha, \beta) \\
\partial X_{1}=-\partial \mathrm{N}\left(K_{1}\right) \stackrel{\cong}{\longrightarrow}, & \left(\mu\left(K_{1}\right), \rho\left(K_{1}\right)\right) \longmapsto(\alpha,-\beta)
\end{array}
$$

Thanks to these identifications, we view $f$ as an orientation-preserving self-homeomorphism of $\mathbb{T}$, and we consider the matrix giving the action of $f$ on $H_{1}(\mathbb{T} ; \mathbb{Z})$ :

$$
\left(\begin{array}{ll}
p & r \\
q & s
\end{array}\right) \quad \text { where } \quad\left\{\begin{array}{l}
f_{*}(\alpha)=p \alpha+q \beta \\
f_{*}(\beta)=r \alpha+s \beta
\end{array}\right.
$$

The following lemma gives a decomposition of this matrix. See [G75, Lemma 4] for a proof. 
Lemma 2.6. Any matrix in $\mathrm{SL}_{2}(\mathbb{Z})$ is a product of matrices $\left(\begin{array}{cc}a & -1 \\ 1 & 0\end{array}\right)$ with $a \in \mathbb{Z}$.

Applying Lemma 2.6 to $\left(\begin{array}{cc}0 & 1 \\ -1 & 0\end{array}\right)\left(\begin{array}{cc}p & r \\ q & s\end{array}\right)$, we get some integers $a_{1}, \ldots, a_{n}$ such that

$$
\left(\begin{array}{cc}
p & r \\
q & s
\end{array}\right)=\left(\begin{array}{cc}
0 & -1 \\
1 & 0
\end{array}\right)\left(\begin{array}{cc}
a_{n} & -1 \\
1 & 0
\end{array}\right) \cdots\left(\begin{array}{cc}
a_{1} & -1 \\
1 & 0
\end{array}\right)
$$

Proposition 2.7. The splice $M$ of $\left(M_{1}, K_{1}\right)$ and $\left(M_{2}, K_{2}\right)$ defined by a homeomorphism $f$, whose matrix (2.4) in homology is decomposed as in (2.5), is the surgered manifold

$$
\left(M_{1} \sharp M_{2}\right)_{K_{1} \sqcup H \sqcup K_{2}}
$$

where $H:=H\left(a_{1}, \ldots, a_{n}\right)$ is the "chain" of Hopf links "clasping" $K_{1}$ and $K_{2}$ as shown below:

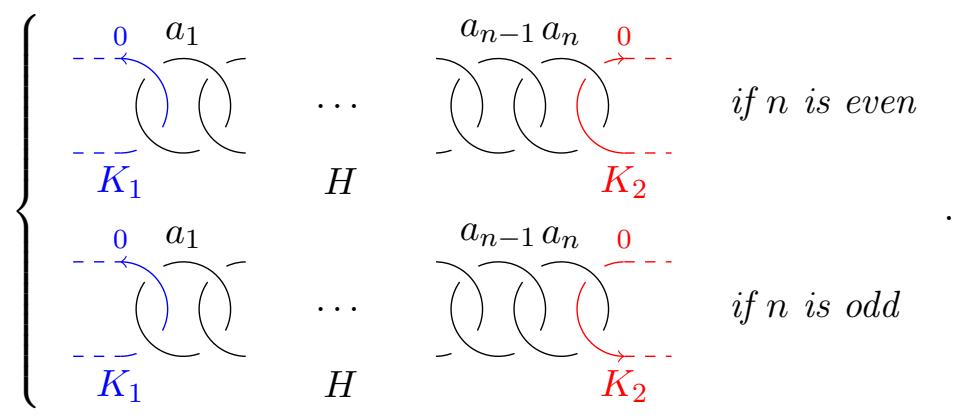

Proof. Let $C_{f}$ be the mapping cylinder of $f$, which we view as a toroidal cobordism. Since $C_{f}$ is determined by its action on homology, Lemma 2.4 implies

$$
C_{f}=\mathcal{L}_{0} \circ \mathcal{L}_{a_{n}} \circ \cdots \circ \mathcal{L}_{a_{1}} .
$$

Viewing $M$ as a cobordism $\emptyset \rightarrow \emptyset, X_{1}$ as a cobordism $\emptyset \rightarrow \mathbb{T}$ and $X_{2}$ as a cobordism $\mathbb{T} \rightarrow \emptyset$, we have $M=X_{2} \circ C_{f} \circ X_{1}$. Hence we conclude with Lemma 2.3 and Lemma 2.5.

\section{SPLICING FORMUla}

In the sequel, we mainly follow the notations of [BL04] which we recall in part. Let $N$ be a rational homology 3-sphere (in short, a $\mathbb{Q} H S$ ) and let $L \subset N$ be a framed knot. We denote by

$$
Z(N, L) \in \mathcal{A}\left(\circlearrowleft_{l}\right)
$$

the Kontsevich-LMO invariant of the pair $(N, L)$. Here the "abstract" oriented 1-manifold $\circlearrowleft$ is labeled with the letter $l$ in order to refer to the unique connected component of $L$ : this convention (lower-to-upper case letters) will be used throughout the text to label connected components. In the absence of knot, $Z(N):=Z(N, \emptyset)$ is the LMO invariant of $N$ which is denoted by $\hat{Z}^{\mathrm{LMO}}(N) \in \mathcal{A}(\emptyset)$ in $[\mathrm{BL04}]^{1}$. In the case of the standard 3-sphere, $Z(L):=$ $Z\left(S^{3}, L\right)$ is the Kontsevich integral of $L$ in the version that is also denoted by $Z(L) \in \mathcal{A}\left(\circlearrowleft_{l}\right)$ in [BL04]. Even if $N \neq S^{3}, Z(N, L)$ will often be abbreviated to $Z(L)$ when $N$ is clear from the context.

Let $\chi: \mathcal{A}\left(*_{X}\right) \rightarrow \mathcal{A}\left(\uparrow_{X}\right)$ be the diagrammatic analogue of the PBW isomorphism, which is defined for any finite set $X$. Denote by

$$
\Omega:=\chi^{-1} Z(\text { unknot }) \in \mathcal{A}(*)
$$

\footnotetext{
${ }^{1}$ This corresponds to the notation $\hat{\Omega}(M)$ in [LMO98] and to $\stackrel{\AA}{A}(M)$ in [BGRT02].
} 
the "symmetrized" value of the unknot. The latter has been found in [BLT03] to be equal to

$$
\Omega=\exp \left(\sum_{m \geq 1} b_{2 m} \omega_{2 m}\right)
$$

where $\omega_{2 m}$ denotes the Jacobi diagram consisting of one "wheel" with $2 m$ "spokes" (as depicted in Figure 3), and the modified Bernoulli numbers

$$
b_{2}=\frac{1}{48}, \quad b_{4}=-\frac{1}{5760}, \quad \text { etc, }
$$

are defined by the formal power series

$$
\begin{gathered}
\sum_{m \geq 1} b_{2 m} X^{2 m}:=\frac{1}{2} \log \left(\frac{\sinh (X / 2)}{X / 2}\right) \in \mathbb{Q}[[X]] . \\
\omega_{2}=-\bigcirc-\quad \omega_{4}=-\omega_{6}=-
\end{gathered}
$$

Figure 3. The diagrams $\omega_{2 m}$

Given a finite set $X$ and $D \in \mathcal{A}\left(*_{X}\right)$, we denote by $\partial_{D}$ (resp. $\langle D,-\rangle$ ) the operator that maps any Jacobi diagram $E$ (having univalent vertices colored by $X$ ) to the sum of all ways of gluing all the $x$-colored vertices of $D$ to some of (resp. to all) the $x$-colored vertices of $E$, for each $x \in X$. In particular, we will need the constant

$$
\omega:=\langle\Omega, \Omega\rangle=\emptyset+\frac{1}{24} \Theta+\left(\begin{array}{c}
\text { trivalent diagrams } \\
\text { with } \geq 6 \text { vertices }
\end{array}\right) \in \mathcal{A}(\emptyset)
$$

It has been proved in [BLT03] that the composition of linear isomorphisms

$$
\mathcal{A}(*) \underset{\Omega}{\stackrel{\partial_{\Omega}}{\longrightarrow}} \mathcal{A}(*) \underset{\simeq}{\stackrel{\chi}{\cong}} \mathcal{A}(\uparrow) \cong \mathcal{A}(\circlearrowleft)
$$

preserves the algebra structures. Thus, as in [BL04], we will work with the wheeled version of the Kontsevich-LMO invariant, namely

$$
Z^{\not}(N, L):=\partial_{\Omega_{l}}^{-1} \chi^{-1}(Z(N, L)) \in \mathcal{A}\left(*_{l}\right) .
$$

Here $\Omega_{l} \in \mathcal{A}\left(*_{l}\right)$ is the same as $\Omega \in \mathcal{A}(*)$ but with each univalent vertex now labeled with $l$. For instance, [BL04, Lemma 3.10] tells us that

$$
Z^{ळ}(\text { unknot })=\omega^{-1} \Omega \in \mathcal{A}(*) .
$$

Let now $M_{1}$ and $M_{2}$ be $\mathbb{Q H S}$. Let $K_{1} \subset M_{1}$ and $K_{2} \subset M_{2}$ be null-homologous knots. We give $K_{i}$ the preferred parallel $\rho\left(K_{i}\right)$, i.e. the one that bounds a surface in the knot exterior $X_{i}=M_{i} \backslash \operatorname{Int}\left(\mathrm{N}\left(K_{i}\right)\right)$. We identify $\partial X_{i}$ with $\mathbb{T}$ as in (2.3), and we consider the splice

$$
M:=X_{1} \bigcup_{f} X_{2}
$$

defined by the homeomorphism $f: \mathbb{T} \rightarrow \mathbb{T}$ that is encoded by four integers $p, q, r, s$ as in (2.4). A Mayer-Vietoris argument shows that $M$ is a $\mathbb{Q H S}$ if and only if $r \neq 0$ (see the proof of Lemma 6.1 below). 
Our main result computes the LMO invariant $Z(M)$ in terms of the wheeled KontsevichLMO invariants $Z^{œ}\left(K_{i}\right)=Z^{œ}\left(M_{i}, K_{i}\right)$. The formula involves Dedekind symbols whose definition is recalled in Appendix A. We also denote by $\theta$ the $\theta$-shaped diagram $\ominus \in \mathcal{A}(\emptyset)$.

Theorem 3.1. Let $K_{1} \subset M_{1}$ and $K_{2} \subset M_{2}$ be null-homologous knots in $\mathbb{Q} H S$, and let $M$ be the splice of $\left(M_{1}, K_{1}\right)$ and $\left(M_{2}, K_{2}\right)$ as described by (3.4) in terms of four integers $p, q, r, s$. If $M$ is also a $\mathbb{Q} H S$, then we have

$$
Z(M)=\omega \exp \left(\frac{\theta}{48}\left(-S\left(\frac{p}{r}\right)+\frac{p+s}{r}\right)\right)\left\langle\left.\partial_{D_{1}}\left(Z^{\not 2}\left(K_{1}\right)\right)\right|_{k_{1} \rightarrow-k_{2} / r}, \partial_{D_{2}}\left(Z^{\not \alpha}\left(K_{2}\right)\right)\right\rangle
$$

where

$$
D_{1}:=\exp \left(-\frac{p}{2 r} \cup^{k_{1} k_{1}}\right) \text { and } D_{2}:=\exp \left(-\frac{s}{2 r} \cup^{k_{2} k_{2}}\right) .
$$

Proof. Choose a matrix decomposition as in (2.5), which leads to $n \geq 1$ integers $a_{1}, \ldots, a_{n}$, and consider the Hopf chain $\widetilde{H}:=H\left(0, a_{1}, \ldots, a_{n}, 0\right) \subset S^{3}$ shown below:

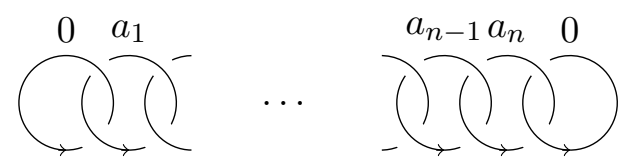

Reading from left to right, the components of $\widetilde{H}$ are denoted $H^{\prime}, H_{1}, \ldots, H_{n}, H^{\prime \prime}$.

Let $\epsilon_{n} K_{2}$ be the knot $K_{2}$ if $n$ is odd, and the knot $K_{2}$ with reversed orientation if $n$ is even. According to Proposition 2.7, $M$ is the result of doing surgery in $M_{1} \sharp M_{2}$ along the link $K_{1} \sqcup H \sqcup \epsilon_{n} K_{2}$ where $H$ is the Hopf chain $H\left(a_{1}, \ldots, a_{n}\right)$ "clasping" positively $K_{1}$ on one side and $\epsilon_{n} K_{2}$ on the other side. This link in $M_{1} \sharp M_{2}=\left(M_{1} \sharp S^{3}\right) \sharp M_{2}$ is obtained from $\left(K_{1} \sqcup \widetilde{H}\right) \sqcup K_{2}$ by doing the connected sum of $K_{1} \subset M_{1}$ with $H^{\prime} \subset S^{3}$, in a first time, and by doing the connected sum of $H^{\prime \prime} \subset M_{1} \sharp S^{3}$ with $\epsilon_{n} K_{2} \subset M_{2}$, in a second time. The way the wheeled Kontsevich integral behaves under connected sums of links is described in [BL04, Lemma 3.9]; the proof given there works as well for the wheeled Kontsevich-LMO invariant. Applying this result, we get

$$
Z^{\not ळ}\left(K_{1} \sqcup H \sqcup \epsilon_{n} K_{2}\right)=\left.\left.\omega^{2} \Omega_{k_{1}}^{-1} \Omega_{k_{2}}^{-1} Z^{\not ்}\left(K_{1}\right) Z^{\not}(\widetilde{H})\right|_{h^{\prime} \rightarrow k_{1}}\right|_{h^{\prime \prime} \rightarrow k_{2}} Z^{\not ळ}\left(\epsilon_{n} K_{2}\right)
$$

where $Z^{\ltimes}\left(K_{1} \sqcup H \sqcup \epsilon_{n} K_{2}\right):=Z^{\ltimes}\left(M_{1} \sharp M_{2}, K_{1} \sqcup H \sqcup \epsilon_{n} K_{2}\right)$. Besides, according to [BL04, Prop. 4.3] which computes the value of $Z^{\not 2}$ on any Hopf chain, we have

$$
\begin{aligned}
Z^{\not ळ}(\widetilde{H})= & \omega^{-1} \exp \left(-\frac{\theta \sum_{i=1}^{n} a_{i}}{48}\right) \prod_{i=1}^{n} \Omega_{h_{i}}^{-1} . \\
& \cdot \exp \left(\underset{h^{\prime} h_{1}}{\cap}+\sum_{i=1}^{n-1} \bigcap_{h_{i} h_{i+1}}+\underset{h_{n} \bigcap_{h^{\prime \prime}}}{\cap}+\frac{1}{2} \sum_{i=1}^{n} a_{i} \underset{h_{i} h_{i}}{\bigcap}\right) .
\end{aligned}
$$

Next, the way the LMO invariant of a $\mathbb{Q H S}$ can be computed from the wheeled Kontsevich integral of a surgery presentation in $S^{3}$ is described in [BL04, Eq. (22)]; the proof given there works as well for a surgery presentation in any $\mathbb{Q} H S$ other than $S^{3}$ — see [BL04, Remark 1.7] in this connection. Applying this result to $M=\left(M_{1} \sharp M_{2}\right)_{K_{1} \sqcup H \sqcup \epsilon_{n} K_{2}}$, we get the diagrammatic 
Gaussian integral

$$
Z(M)=\exp \left(\frac{\theta \zeta(\Lambda)}{16}\right) \int Z^{\not 2}\left(K_{1} \sqcup H \sqcup \epsilon_{n} K_{2}\right) \Omega_{k_{1}} \Omega_{k_{2}} \prod_{i=1}^{n} \Omega_{h_{i}} \mathrm{~d} k_{1} \mathrm{~d} h_{1} \cdots \mathrm{d} h_{n} \mathrm{~d} k_{2},
$$

where $\zeta(\Lambda)$ denotes the signature of the linking matrix $\Lambda$ of $K_{1} \sqcup H \sqcup \epsilon_{n} K_{2}$ in $M_{1} \sharp M_{2}$. Note that $\Lambda$ is the tridiagonal matrix

$$
\Lambda=\left(\begin{array}{cccccc}
0 & 1 & 0 & \cdots & 0 & 0 \\
1 & a_{1} & 1 & \cdots & 0 & 0 \\
0 & 1 & a_{2} & \cdots & 0 & 0 \\
\vdots & \vdots & \vdots & \ddots & \vdots & \vdots \\
0 & 0 & 0 & \cdots & a_{n} & 1 \\
0 & 0 & 0 & \cdots & 1 & 0
\end{array}\right)
$$

Combining this with (3.6) and (3.7), we deduce that

$$
\begin{aligned}
& Z(M)=\omega \exp \left(\frac{\theta(3 \zeta(\Lambda)-\operatorname{tr}(\Lambda))}{48}\right) \int Z^{\not}\left(K_{1}\right) Z^{ळ}\left(\epsilon_{n} K_{2}\right) \cdot \\
& \cdot \exp \left(\underset{k_{1} h_{1}}{\cap}+\sum_{i=1}^{n-1} \underset{h_{i} h_{i+1}}{\cap}+\underset{h_{n} k_{2}}{\cap}+\frac{1}{2} \sum_{i=1}^{n} a_{i} \underset{h_{i} h_{i}}{\cap}\right) \mathrm{d} k_{1} \mathrm{~d} h_{1} \cdots \mathrm{d} h_{n} \mathrm{~d} k_{2} .
\end{aligned}
$$

Since $K_{1}$ is endowed with its preferred parallel, $Z^{\not}\left(K_{1}\right)$ has no strut; similarly $Z^{\ltimes}\left(\epsilon_{n} K_{2}\right)$ has no strut. Thus, the "strut" part of the above integrand is fully given by the "exp" part that is apparent there. To compute this Gaussian integral, we need to invert the tridiagonal matrix $\Lambda$ but, knowing that $Z^{œ}\left(K_{1}\right) Z^{\natural}\left(\epsilon_{n} K_{2}\right)$ has no univalent vertex colored by $h_{1}, \ldots, h_{n}$, we only need to determine the four corners of $\Lambda^{-1}$. To this purpose, we compute the $2 \times 2$ matrix associated to the tridiagonal matrix $\Lambda$ (see Appendix A):

$$
\begin{aligned}
& \left(\begin{array}{cc}
0 & -1 \\
1 & 0
\end{array}\right) \cdot\left(\begin{array}{cc}
0 & -1 \\
1 & 0
\end{array}\right)\left(\begin{array}{cc}
a_{1} & -1 \\
1 & 0
\end{array}\right) \cdots\left(\begin{array}{cc}
a_{n} & -1 \\
1 & 0
\end{array}\right)\left(\begin{array}{cc}
0 & -1 \\
1 & 0
\end{array}\right) \\
= & \left(\begin{array}{cc}
0 & -1 \\
1 & 0
\end{array}\right) \cdot\left(\begin{array}{cc}
0 & -1 \\
1 & 0
\end{array}\right) \cdot\left(\begin{array}{cc}
1 & 0 \\
0 & -1
\end{array}\right) \cdot\left(\begin{array}{cc}
a_{1} & 1 \\
-1 & 0
\end{array}\right) \cdots\left(\begin{array}{cc}
a_{n} & 1 \\
-1 & 0
\end{array}\right)\left(\begin{array}{cc}
0 & 1 \\
-1 & 0
\end{array}\right) \cdot\left(\begin{array}{cc}
1 & 0 \\
0 & -1
\end{array}\right) \\
\stackrel{(2.5)}{=} & \left(\begin{array}{cc}
0 & -1 \\
1 & 0
\end{array}\right) \cdot\left(\begin{array}{cc}
0 & -1 \\
1 & 0
\end{array}\right) \cdot\left(\begin{array}{cc}
1 & 0 \\
0 & -1
\end{array}\right) \cdot\left(\begin{array}{ll}
p & r \\
q & s
\end{array}\right)^{T} \cdot\left(\begin{array}{cc}
1 & 0 \\
0 & -1
\end{array}\right)=\left(\begin{array}{cc}
-p & q \\
r & -s
\end{array}\right)
\end{aligned}
$$

and we deduce from Proposition A.1 that

$$
\Lambda^{-1}=\left(\begin{array}{ccccc}
p / r & ? & \cdots & ? & (-1)^{n+1} / r \\
? & ? & \cdots & ? & ? \\
\vdots & \vdots & \ddots & \vdots & \vdots \\
? & ? & \cdots & ? & ? \\
(-1)^{n+1} / r & ? & \cdots & ? & s / r
\end{array}\right)
$$

Therefore, performing Gaussian integration, we get

$$
\begin{aligned}
Z(M)= & \omega \exp \left(\frac{\theta(3 \zeta(\Lambda)-\operatorname{tr}(\Lambda))}{48}\right) \cdot \\
& \cdot\left\langle\exp \left(-\frac{p}{2 r} \cup^{k_{1} k_{1}}+\frac{(-1)^{n}}{r} \cup^{k_{1}}-\frac{s}{2 r} \cup^{k_{2}}\right), Z^{k_{2}}\left(K_{1}\right) Z^{\infty}\left(\epsilon_{n} K_{2}\right)\right\rangle
\end{aligned}
$$


and, since $Z^{\not \alpha}\left(-K_{2}\right)$ is obtained from $Z^{\not}\left(K_{2}\right)$ by the change $k_{2} \rightarrow-k_{2}$, this is equivalent to

$$
\begin{aligned}
Z(M)= & \omega \exp \left(\frac{\theta(3 \zeta(\Lambda)-\operatorname{tr}(\Lambda))}{48}\right) \cdot \\
& \cdot\left\langle\exp \left(-\frac{p}{2 r} \cup^{k_{1} k_{1}}-\frac{1}{r} \bigcup^{k_{1} k_{2}}-\frac{s}{2 r} \cup^{k_{2} k_{2}}\right), Z^{\not \infty}\left(K_{1}\right) Z^{\not \infty}\left(K_{2}\right)\right\rangle
\end{aligned}
$$

whatever the parity of $n$ is. Using now Theorem A.3, we obtain

$$
\begin{aligned}
Z(M)= & \omega \exp \left(\frac{\theta}{48}\left(-S\left(\frac{p}{r}\right)+\frac{p+s}{r}\right)\right) . \\
& \cdot\left\langle\exp \left(-\frac{p}{2 r} \cup^{k_{1} k_{1}}-\frac{1}{r} \cup^{k_{1} k_{2}}-\frac{s}{2 r} \cup^{k_{2}}\right), Z^{\not \infty}\left(K_{1}\right) Z^{\not \infty}\left(K_{2}\right)\right\rangle .
\end{aligned}
$$

Using the notation (3.5), this is equivalent to

$$
Z(M)=\omega \exp \left(\frac{\theta}{48}\left(-S\left(\frac{p}{r}\right)+\frac{p+s}{r}\right)\right)\left\langle\exp \left(-\frac{1}{r} \cup^{k_{1} k_{2}}\right), \partial_{D_{1}}\left(Z^{\rightsquigarrow}\left(K_{1}\right)\right) \partial_{D_{2}}\left(Z^{œ}\left(K_{2}\right)\right)\right\rangle
$$

and the conclusion easily follows.

Example 3.2. When the splice of $\left(M_{1}, K_{1}\right)$ and $\left(M_{2}, K_{2}\right)$ is standard, Theorem 3.1 simplifies as follows. Then $\mu\left(K_{1}\right)$ and $\rho\left(K_{1}\right)$ are glued along $\rho\left(K_{2}\right)$ and $\mu\left(K_{2}\right)$, respectively: thus $p=s=0$ and $q=-r=1$. Hence, by identifying the symbols $k_{1}$ and $k_{2}$, we get

$$
Z(M)=\omega\left\langle Z^{\mathfrak{\infty}}\left(K_{1}\right), Z^{\mathfrak{\infty}}\left(K_{2}\right)\right\rangle \text {. }
$$

Bar-Natan and Lawrence developed their techniques (which we have intensively used in the proof of Theorem 3.1) to prove a rational surgery formula for the LMO invariant [BL04, Eq. (23)]. We now explain how their formula - in the case of a rational surgery along a single knot - is recovered from Theorem 3.1.

Corollary 3.3 (Bar-Natan \& Lawrence). Let $L \subset N$ be a null-homologous knot in a $\mathbb{Q} H S$, with framing given by the preferred parallel. Let $r, s$ be non-zero coprime integers, and let $M$ be obtained from $N$ by an $(r / s)$-surgery along $L$. Then

$$
Z(M)=\exp \left(\frac{\theta}{48}\left(-S\left(\frac{s}{r}\right)+\frac{s}{r}\right)\right)\left\langle\Omega_{l / r}, \partial_{D}\left(Z^{\not}(L)\right)\right\rangle \quad \text { with } D:=\exp \left(-\frac{s}{2 r} \stackrel{l}{ }^{l}\right)
$$

and the latter identity is equivalent to [BL04, Eq. (23)].

Proof. We consider $\left(M_{1}, K_{1}\right):=\left(S^{3}\right.$, unknot $),\left(M_{2}, K_{2}\right):=(N, L)$ and the integers $p, q$ are chosen so that $p s-q r=1$. Then, the corresponding splice is the 3 -manifold $M$. Recall from ( 3.3) that $Z^{\infty}\left(K_{1}\right)=\omega^{-1} \Omega_{k_{1}}$. Hence, in this particular situation, Theorem 3.1 gives

$$
Z(M)=\exp \left(\frac{\theta}{48}\left(-S\left(\frac{p}{r}\right)+\frac{p+s}{r}\right)\right)\left\langle\left.\partial_{D_{1}}\left(\Omega_{k_{1}}\right)\right|_{k_{1} \rightarrow-k_{2} / r}, \partial_{D_{2}}\left(Z^{\not \infty}\left(K_{2}\right)\right)\right\rangle
$$

where

$$
D_{1}:=\exp \left(-\frac{p}{2 r} \stackrel{k_{1} k_{1}}{\cup}\right) \text { and } D_{2}:=\exp \left(-\frac{s}{2 r}{\stackrel{k}{k_{2}}}^{k_{2}}\right) .
$$

Besides, recall from [BL04, Cor. 3.4] that, for any $\alpha \in \mathbb{Q}$, we have

$$
\partial_{E}(\Omega)=\exp \left(\frac{\alpha \theta}{48}\right) \Omega \in \mathcal{A}(*) \quad \text { where } E:=\exp \left(\frac{\alpha}{2} \cup\right) \text {. }
$$


In particular, $\partial_{D_{1}} \Omega_{k_{1}}=\exp \left(-\frac{p}{r} \frac{\theta}{48}\right) \Omega_{k_{1}}$. Hence, using that $\Omega$ only shows diagrams with an even number of univalent vertices (see (3.1)) and that $S(p / r)=S(s / r)$ (since $p \equiv s^{-1}$ $\bmod r$ ), we get

$$
Z(M)=\exp \left(\frac{\theta}{48}\left(-S\left(\frac{s}{r}\right)+\frac{s}{r}\right)\right)\left\langle\Omega_{l / r}, \partial_{D}\left(Z^{\not x}(L)\right)\right\rangle .
$$

We now check the equivalence between this formula and [BL04, Eq. (23)], which states (in the case of $N=S^{3}$ ) that

$$
Z(M)=\exp \left(\frac{\theta}{48}\left(3 \operatorname{sign}\left(\frac{r}{s}\right)+S\left(\frac{r}{s}\right)-\frac{r}{s}\right)\right)\left\langle\exp \left(\frac{-s}{2 r} \bigcup^{l}\right), Z^{\not \infty}(L) \Omega_{l / s}\right\rangle .
$$

Note that

$$
\left\langle\exp \left(\frac{-s}{2 r} \stackrel{l}{l}^{l}\right), Z^{\infty}(L) \Omega_{l / s}\right\rangle=\left\langle\left.\partial_{D}\left(\Omega_{l / s}\right)\right|_{l \rightarrow-s l / r}, \partial_{D}\left(Z^{\infty}(L)\right)\right\rangle ;
$$

besides, using ( 3.9$)$ one more time, we obtain

$$
\partial_{D}\left(\Omega_{l / s}\right)=\exp \left(-\frac{\theta}{48} \frac{1}{r s}\right) \Omega_{l / s}
$$

hence we deduce that

$$
\left\langle\exp \left(\frac{-s}{2 r} \cup^{l}\right), Z^{\infty}(L) \Omega_{l / s}\right\rangle=\exp \left(-\frac{\theta}{48} \frac{1}{r s}\right)\left\langle\Omega_{l / r}, \partial_{D}\left(Z^{œ}(L)\right)\right\rangle .
$$

Then the equivalence between ( 3.10) and ( 3.11) follows from the reciprocity law ( A.6) for Dedekind symbols.

Example 3.4. Think of the lens space $L(r, s)$ as $S^{3}$ surgered along the $(r / s)$-framed unknot. Then, a direct application of Corollary 3.3 using ( 3.3$)$ and ( 3.9), again, gives

$$
Z(L(r, s))=\omega^{-1} \exp \left(-\frac{\theta}{48} S\left(\frac{s}{r}\right)\right)\left\langle\Omega_{l / r}, \Omega_{l}\right\rangle .
$$

This formula is easily seen to be equivalent to [BL04, Prop. 5.1].

\section{LOW DEGREE FORMULAS}

In this section, we apply Theorem 3.1 to get splicing formulas for the lowest degree terms of the LMO invariant. In the sequel, the degree of a Jacobi diagram refers to its internal degree, $i e$ its number of trivalent vertices, and the symbol " $\equiv_{n}$ " stands for an equality "up to terms of degree at least $n "$.

According to [LMO98, Prop. 5.3], the LMO invariant of any $\mathbb{Q H S} M$ can be written as follows up to degree 5 :

$$
Z(M) \equiv_{6} \emptyset+\frac{1}{4} \lambda_{\mathrm{W}}(M) \ominus+\lambda_{2}(M) \Theta+\frac{1}{32} \lambda_{\mathrm{W}}(M)^{2} \ominus \ominus .
$$

Here $\lambda_{\mathrm{W}}(M) \in \mathbb{Q}$ is the Casson-Walker invariant of $M$ as normalized in [W92], and $\lambda_{2}(M) \in \mathbb{Q}$ is a finite-type invariant of degree 4 (which, up to a multiplicative constant, is the second term of the Ohtsuki series of $M[\mathrm{O} 96 \mathrm{~b}])$.

In [F93, Cor. 1.2], Fujita gives a formula for the Casson-Walker invariant of a $\mathbb{Q} H S$ obtained as the splice of two knots in integral homology 3-spheres. The next proposition generalizes 
his result to the splice of null-homologous knots in $\mathbb{Q H S}$. Given a null-homologous knot $K$ in a $\mathbb{Q H S} M$, denote by

$$
\Delta_{K}(t) \in \mathbb{Q}\left[t^{ \pm 1}\right]
$$

its Alexander polynomial normalized so that $\Delta_{K}\left(t^{-1}\right)=\Delta_{K}(t)$ and $\Delta_{K}(1)=1$ (see [M12a, Theorem 1.5]).

Proposition 4.1 (Fujita). Let $K_{1} \subset M_{1}$ and $K_{2} \subset M_{2}$ be null-homologous knots in $\mathbb{Q} H S$ and let $M$ be the splice of $\left(M_{1}, K_{1}\right)$ and $\left(M_{2}, K_{2}\right)$ described by (3.4) in terms of four integers $p, q, r, s$. If $M$ is also a $\mathbb{Q} H S$, then

$$
\lambda_{\mathrm{W}}(M)=\lambda_{\mathrm{W}}\left(M_{1}\right)+\lambda_{\mathrm{W}}\left(M_{2}\right)-\frac{1}{12} S\left(\frac{p}{r}\right)+\frac{p}{r} \Delta_{K_{1}}^{\prime \prime}(1)+\frac{s}{r} \Delta_{K_{2}}^{\prime \prime}(1) .
$$

Example 4.2. For a standard splicing, we have $\lambda_{\mathrm{W}}(M)=\lambda_{\mathrm{W}}\left(M_{1}\right)+\lambda_{\mathrm{W}}\left(M_{2}\right)$. This additivity formula for $\lambda_{\mathrm{W}}$ was obtained in [BN90, FN88].

Remark 4.3. In the case $\left(M_{1}, K_{1}\right):=\left(S^{3}\right.$, unknot), Proposition 4.1 specializes to Walker's rational surgery formula for $\lambda_{\mathrm{W}}$ - see [W92, Prop. (6.2)].

To express the splicing formula for $\lambda_{2}$, we need the following invariant of a null-homologous knot $K$ in a $\mathbb{Q} H S M$ : let $v(K)$ be the coefficient of $\bigcup$ in $\chi^{-1} Z(K)$ where $Z(K)=Z(M, K)$ is the Kontsevich-LMO invariant. When $M=S^{3}$, we have $v(K)=-\frac{1}{24} j_{3}(K)$ where $j_{3}(K)$ is the coefficient of $h^{3}$ in the Jones polynomial $V_{K}(t)$ evaluated at $t:=e^{h}$ (see [117, Lemma 2.1]).

Proposition 4.4. Let $K_{1} \subset M_{1}$ and $K_{2} \subset M_{2}$ be null-homologous knots in $\mathbb{Q} H S$ and let $M$ be the splice of $\left(M_{1}, K_{1}\right)$ and $\left(M_{2}, K_{2}\right)$ described by (3.4) in terms of four integers $p, q, r, s$. If $M$ is also a $\mathbb{Q} H S$, then

$$
\begin{aligned}
\lambda_{2}(M)= & \lambda_{2}\left(M_{1}\right)+\lambda_{2}\left(M_{2}\right)+\frac{1}{1152}\left(\frac{1}{r^{2}}-1\right) \\
& +\frac{1}{96}\left(1-\frac{1}{r^{2}}\right)\left(\Delta_{K_{1}}^{\prime \prime}(1)+\Delta_{K_{2}}^{\prime \prime}(1)\right)+\frac{9}{16} \frac{p^{2}}{r^{2}} \Delta_{K_{1}}^{\prime \prime}(1)+\frac{9}{16} \frac{s^{2}}{r^{2}} \Delta_{K_{2}}^{\prime \prime}(1) \\
& +\frac{7}{32} \frac{p^{2}}{r^{2}}\left(\Delta_{K_{1}}^{\prime \prime}(1)\right)^{2}+\frac{7}{32} \frac{s^{2}}{r^{2}}\left(\Delta_{K_{2}}^{\prime \prime}(1)\right)^{2}+\frac{1}{8 r^{2}} \Delta_{K_{1}}^{\prime \prime}(1) \Delta_{K_{2}}^{\prime \prime}(1) \\
& -\frac{5}{96} \frac{p^{2}}{r^{2}} \Delta_{K_{1}}^{(4)}(1)-\frac{5}{96} \frac{s^{2}}{r^{2}} \Delta_{K_{2}}^{(4)}(1)-\frac{p}{r} v\left(K_{1}\right)-\frac{s}{r} v\left(K_{2}\right) .
\end{aligned}
$$

Example 4.5. For a standard splicing, we have $\lambda_{2}(M)=\lambda_{2}\left(M_{1}\right)+\lambda_{2}\left(M_{2}\right)+\frac{1}{8} \Delta_{K_{1}}^{\prime \prime}(1) \Delta_{K_{2}}^{\prime \prime}(1)$. We note that the same non-additivity formula is satisfied for the "SU(3) Casson invariant" in the case when $K_{1}$ and $K_{2}$ are torus knots in $S^{3}$ [BH09], but the relation between this gauge-theoretical invariant and finite-type invariants does not seem to be known yet.

Remark 4.6. In the case $\left(M_{1}, K_{1}\right):=\left(S^{3}\right.$, unknot), Proposition 4.4 specializes to Ito's rational surgery formula for $\lambda_{2}$ - see [117, Theorem 1.2].

To deduce from Theorem 3.1 the above splicing formulas for $\lambda_{\mathrm{W}}$ and $\lambda_{2}$, we need first to determine the low-degree terms of the wheeled Kontsevich-LMO invariant $Z^{\text {k }}$. The following result of Kricker (consequence of [K00, Theorem 1.0.8]) gives the "one-loop" part of $Z^{\not \ltimes}$. Kricker indeed works with a knot in an integral homology 3-sphere, but the whole article adapts in the case of a null-homologous knot in a $\mathbb{Q H S}$, with the above normalization of the Alexander polynomial. 
Theorem 4.7 (Kricker). Let $K$ be a null-homologous knot in a $\mathbb{Q} H S$, with framing given by the preferred parallel. Then the Kontsevich-LMO invariant of $K$ writes $\chi^{-1} Z(K)=$ $\mathrm{Wh}(K) \exp (R)$ where $R$ is a $\mathbb{Q}$-linear combination of connected Jacobi diagrams with at least two loops and

$$
\mathrm{Wh}(K):=\Omega \exp \left(-\left.\frac{1}{2} \log \left(\Delta_{K}\left(e^{h}\right)\right)\right|_{h^{2 m} \rightarrow \omega_{2 m}}\right) .
$$

In the computations to come, it will be more convenient to use the Conway normalization

$$
\nabla_{K}(z)=1+\sum_{m>0} a_{2 m}(K) z^{2 m}
$$

of the Alexander polynomial of a knot $K$, given by $\nabla_{K}\left(t-t^{-1}\right)=\Delta_{K}\left(t^{2}\right)$. In particular, derivating four times this equality gives

$$
\Delta_{K}^{\prime \prime}(1)=2 a_{2}(K) \quad \text { and } \quad \Delta_{K}^{(4)}(1)=24\left(a_{2}(K)+a_{4}(K)\right) .
$$

Lemma 4.8. Let $K$ be a null-homologous knot in a $\mathbb{Q} H S M$, and let $a_{2 i}:=a_{2 i}(K)$ with $i \geq 1$ be the coefficients of the Alexander-Conway polynomial of $K$. Then

$$
\begin{aligned}
\chi^{-1} Z(K) \equiv_{6} \emptyset & +\frac{\lambda_{\mathrm{W}}(M)}{4} \bigcirc+\lambda_{2}(M) \ominus+\frac{\lambda_{\mathrm{W}}(M)^{2}}{32} \bigcirc \bigcirc+\left(b_{2}-\frac{1}{2} a_{2}\right) \bigodot^{k} \\
& +\frac{\lambda_{\mathrm{W}}(M)}{4}\left(b_{2}-\frac{1}{2} a_{2}\right) \ominus \bigcirc^{k}+\frac{1}{2}\left(b_{2}-\frac{1}{2} a_{2}\right)^{2} \bigcirc^{k} \bigcirc^{k} \\
& +v(K) \oslash^{k}+\left(b_{4}-\frac{1}{24} a_{2}+\frac{1}{4} a_{2}^{2}-\frac{1}{2} a_{4}\right) \bigodot_{k}^{k}
\end{aligned}
$$

where $b_{2}, b_{4}$ are the first modified Bernoulli numbers ( 3.2 ).

Proof. It is well-known (and easily verified from the AS and IHX relations) that the following diagrams constitute a basis of the "strut-less" and "connected" part of $\mathcal{A}\left(*_{k}\right)$ up to degree 5 :

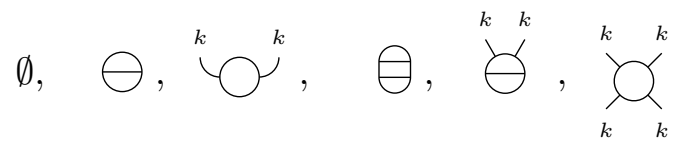

Hence, by the group-like property of $\chi^{-1} Z(K)$ and the fact that its purely-trivalent part reduces to $Z(M)$, it suffices to show that its connected one-loop part equals

$$
\left(b_{2}-\frac{1}{2} a_{2}\right) \Upsilon^{k}+\left(b_{4}-\frac{1}{24} a_{2}+\frac{1}{4} a_{2}^{2}-\frac{1}{2} a_{4}\right){\underset{k}{k}}_{\bigcirc_{k}^{k}}^{k}
$$

up to degree 5 . We have

$$
\Delta_{K}\left(e^{h}\right)=\nabla_{K}\left(e^{\frac{h}{2}}-e^{-\frac{h}{2}}\right)=1+a_{2} h^{2}+\left(\frac{a_{2}}{12}+a_{4}\right) h^{4}+(\operatorname{deg} \geq 6)
$$

thus

$$
\log \left(\Delta_{K}\left(e^{h}\right)\right)=a_{2} h^{2}+\left(\frac{a_{2}}{12}-\frac{a_{2}^{2}}{2}+a_{4}\right) h^{4}+(\operatorname{deg} \geq 6)
$$

and we deduce that

$$
\log \mathrm{Wh}(K) \equiv_{6}\left(b_{2}-\frac{1}{2} a_{2}\right) \omega_{2}+\left(b_{4}-\frac{1}{24} a_{2}+\frac{1}{4} a_{2}^{2}-\frac{1}{2} a_{4}\right) \omega_{4} .
$$


We conclude thanks to Theorem 4.7 .

Lemma 4.9. Let $K$ be a null-homologous knot in a $\mathbb{Q} H S M$, and let $a_{2 i}:=a_{2 i}(K)$ with $i \geq 1$ be the coefficients of the Alexander-Conway polynomial of $K$. Then

$$
\begin{aligned}
& Z^{\not}(K) \equiv_{6} \emptyset+\frac{\lambda_{\mathrm{W}}(M)}{4} \ominus+\left(a_{2} b_{2}-2 b_{2}^{2}+\lambda_{2}(M)\right) \ominus+\frac{\lambda_{\mathrm{W}}(M)^{2}}{32} \ominus \ominus \\
& +\frac{\lambda_{\mathrm{W}}(M)}{4}\left(b_{2}-\frac{1}{2} a_{2}\right) \bigcirc \bigcirc^{k}+\frac{1}{2}\left(b_{2}-\frac{1}{2} a_{2}\right)^{2} \bigcirc^{k} \bigcirc^{k}
\end{aligned}
$$

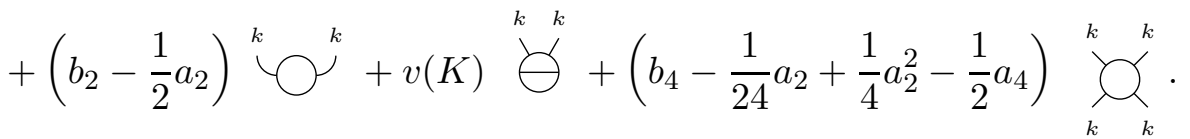

Proof. We have $\Omega^{-1} \equiv_{3} \emptyset-b_{2} \omega_{2}$. Since $\chi^{-1} Z(K)$ is strut-less, we have

$$
Z^{\ltimes}(K)=\partial_{\Omega^{-1}}\left(\chi^{-1} Z(K)\right) \equiv_{6} \chi^{-1} Z(K)-2 b_{2}\left(\text { coef. of } \omega_{2} \text { in } \chi^{-1} Z(K)\right)
$$

and we conclude with Lemma 4.8.

We have now gathered all we need to prove the splicing formulas for $\lambda_{\mathrm{W}}$ and $\lambda_{2}$.

Proof of Proposition 4.1. In the equality of Theorem 3.1, the coefficient of $\theta$ in the left hand side is $\lambda_{\mathrm{W}}(M) / 4$, while the coefficient of $\theta$ in the right hand side is

$$
\begin{aligned}
& \frac{1}{48}\left(\frac{s+p}{r}-S\left(\frac{p}{r}\right)\right)+\frac{1}{4} \lambda_{\mathrm{W}}\left(M_{1}\right)+\frac{1}{4} \lambda_{\mathrm{W}}\left(M_{2}\right) \\
& -\frac{p}{r}\left(b_{2}-\frac{1}{2} a_{2}\left(K_{1}\right)\right)-\frac{s}{r}\left(b_{2}-\frac{1}{2} a_{2}\left(K_{2}\right)\right) .
\end{aligned}
$$

We conclude by substituting the values (3.2) and (4.2).

Proof of Proposition 4.4. We compute the coefficient of $\Theta$ in the right hand side of the equality in Theorem 3.1. First of all, the contribution of $\omega$ is $2 b_{2}^{2}$. Next, $\partial_{D_{i}}\left(Z^{\infty}\left(K_{i}\right)\right)$ contributes in two ways to this coefficient: there is an "invidual" contribution through its $\Theta$ part, and there is a "mutual" contribution through its $\bigcirc$ part. On the one hand, the individual contribution of $\partial_{D_{i}}\left(Z^{\not}\left(K_{i}\right)\right)$ is

$$
a_{2} b_{2}-2 b_{2}^{2}+\lambda_{2}\left(M_{i}\right)-\frac{t}{r} v\left(K_{i}\right)+\frac{t^{2}}{r^{2}}\left(b_{2}-\frac{1}{2} a_{2}\right)^{2}+\frac{5}{2} \frac{t^{2}}{r^{2}}\left(b_{4}-\frac{1}{24} a_{2}+\frac{1}{4} a_{2}^{2}-\frac{1}{2} a_{4}\right)
$$

where $a_{\ell}:=a_{\rho}\left(K_{i}\right), t:=p$ if $i=1$ and $t:=s$ if $i=2$ (to compute the sub-contribution resulting from the $\bigcirc$ term of $Z^{\infty}\left(K_{i}\right)$, we use an IHX relation that gives $\Theta=2 \mathbb{\Delta}$ ). On the other hand, the "mutual" contribution of $\partial_{D_{1}}\left(Z^{\not}\left(K_{1}\right)\right)$ and $\partial_{D_{2}}\left(Z^{\not}\left(K_{2}\right)\right)$ is

$$
\frac{2}{r^{2}}\left(b_{2}-\frac{1}{2} a_{2}\left(K_{1}\right)\right)\left(b_{2}-\frac{1}{2} a_{2}\left(K_{2}\right)\right) .
$$

Summing all these contributions and substituting the values (3.2) and (4.2), we obtain the desired formula for $\lambda_{2}(M)$. 


\section{Splicing And SATEllite operations}

The following generalization of Theorem 3.1 is proved exactly in the same way.

Theorem 5.1. Let $K_{1} \subset M_{1}$ and $K_{2} \subset M_{2}$ be null-homologous knots in $\mathbb{Q} H S$ and let $M$ be the splice of $\left(M_{1}, K_{1}\right)$ and $\left(M_{2}, K_{2}\right)$ as described by (3.4) in terms of four integers $p, q, r, s$, assuming that $r \neq 0$. For each $i \in\{1,2\}$, let $L_{i} \subset M_{i}$ be a framed link disjoint from $K_{i}$ and let

$$
Z^{\bowtie} k_{i}\left(M_{i}, K_{i} \sqcup L_{i}\right):=\partial_{\Omega_{k_{i}}}^{-1} \chi_{k_{i}}^{-1} Z\left(M_{i}, K_{i} \sqcup L_{i}\right)
$$

be the Kontsevich-LMO invariant of $\left(M_{i}, K_{i} \sqcup L_{i}\right)$ "wheeled" at $K_{i}$. Then

$$
\begin{aligned}
Z\left(M, L_{1} \sqcup L_{2}\right)= & \omega \exp \left(\frac{\theta}{48}\left(-S\left(\frac{p}{r}\right)+\frac{p+s}{r}\right)\right) \cdot \\
& \cdot\left\langle\left.\partial_{D_{1}}\left(Z^{\ltimes k_{1}}\left(M_{1}, K_{1} \sqcup L_{1}\right)\right)\right|_{k_{1} \rightarrow-k_{2} / r}, \partial_{D_{2}}\left(Z^{\bowtie k_{2}}\left(M_{2}, K_{2} \sqcup L_{2}\right)\right)\right\rangle
\end{aligned}
$$

where

$$
D_{1}:=\exp \left(-\frac{p}{2 r} \cup^{k_{1} k_{1}}\right) \text { and } D_{2}:=\exp \left(-\frac{s}{2 r} \cup^{k_{2} k_{2}}\right) .
$$

Example 5.2. For a standard splice, identifying the symbols $k_{1}$ and $k_{2}$, we get

$$
Z\left(M, L_{1} \sqcup L_{2}\right)=\omega\left\langle Z^{\ltimes k_{1}}\left(M_{1}, K_{1} \sqcup L_{1}\right), Z^{\ltimes k_{2}}\left(M_{2}, K_{2} \sqcup L_{2}\right)\right\rangle .
$$

Let $P$ be a framed link in the solid torus $S^{1} \times D^{2}$ and let $L$ be a framed knot in an oriented 3-manifold $N$. We identify $S^{1} \times D^{2}$ with the tubular neighborhood $\mathrm{N}(L)$ so that $S^{1} \times\{0\}$ and $S^{1} \times\{1\}$ correspond to the knot $L$ and its parallel $\rho(L)$, respectively. The $P$-satellite of $L$ is the image of $P$ in $N$ by this identification $S^{1} \times D^{2} \cong \mathrm{N}(L)$; this framed link is denoted by

$$
L_{P} \subset N \text {. }
$$

It is well-known that $L_{P}$ can be constructed by splicing: identify $S^{1} \times D^{2}$ with the exterior of the unknot $U \subset S^{3}$ so that both $S^{1} \times\{0\}$ and $S^{1} \times\{1\}$ are oriented meridians of $U$; then the standard splice of $(N, L)$ and $\left(S^{3}, U\right)$ is a copy of $N$ where $P \subset S^{3} \backslash \mathrm{N}(U)$ corresponds to $L_{P}$. Hence the following is an immediate consequence of Theorem 5.1 in the simplified version of (5.1).

Corollary 5.3. Let $L \subset N$ be a null-homologous knot in a $\mathbb{Q} H S$ (with framing given by the preferred parallel), and let $P \subset S^{1} \times D^{2}$ be a framed link. Then, by identifying the symbols $l$ and $u$, we have

$$
Z\left(N, L_{P}\right)=\omega\left\langle Z^{\not \infty}(N, L), Z^{\not u}\left(S^{3}, U \sqcup P\right)\right\rangle .
$$

We now derive from the "satellite formula" ( 5.2) a generalization of a formula of Suetsugu [S96]. The latter involves the extension of the Kontsevich integral to framed links in a solid torus [S96] or, equivalently, in a thickened annulus [AMR98]. For an $n$-component framed link $P \subset S^{1} \times D^{2}$, this invariant

$$
Z^{\circ}(P) \in \mathcal{A}^{\mathbf{0}}\left(\circlearrowleft^{1} \cdots \circlearrowleft^{n}\right)
$$

is valued in the space generated by homotopy classes of immersions from chord diagrams on $\circlearrowleft^{1} \cdots \circlearrowleft^{n}$ to the annulus, modulo the $4 \mathrm{~T}$ relation. 
Let $D$ be a Jacobi diagram on $\circlearrowleft$ (possibly with some univalent vertices colored by a finite set $X$ ) and let $E$ be a chord diagram on $\circlearrowleft^{1} \cdots \circlearrowleft^{n}$ in the annulus. One can produce from $D$ and $E$ a linear combination of Jacobi diagrams

$$
\left.D\right|_{\circlearrowleft \rightarrow E}
$$

on $\circlearrowleft^{1} \cdots \circlearrowleft^{n}$ (possibly with some univalent vertices colored by $X$ ) by the following procedure: (1) thicken the 1-manifold $\circlearrowleft$ in $D$ to an annulus, replacing every univalent vertex of $D$ by a box directed in the same direction as $\circlearrowleft$; (2) put $E$ inside this annulus; (3) apply the "box notation" below:

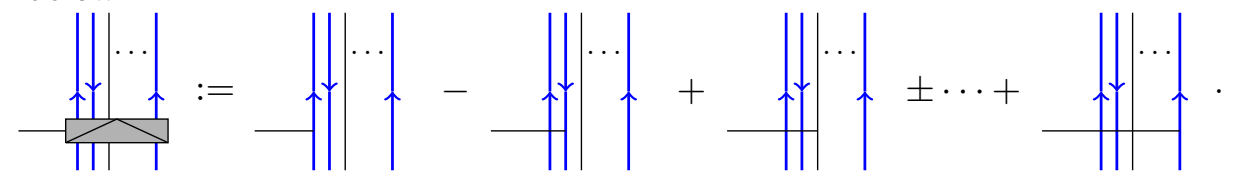

Here, some pieces of chords of $E$ and some arcs of the 1-manifold $\circlearrowleft^{1} \ldots \circlearrowleft^{n}$ (which are shown in bold blue) go through the box, and each of them contributes to one summand in the box notation; an arc contributes with a + or a -, depending on the compatibility of its orientation with the direction of the box; a piece of chord always contributes with a + , the orientation of the new trivalent vertex being determined by the direction of the box. Of course, for step (2) to make sense, it is necessary to assume that (after a small homotopy) no univalent vertex of $E$ (ie no extremity of any chord) is contained in one of the boxes. That the resulting operation

$$
\mathcal{A}\left(\circlearrowleft, *_{X}\right) \times \mathcal{A}^{\circ}\left(\circlearrowleft^{1} \cdots \circlearrowleft^{n}\right) \longrightarrow \mathcal{A}\left(\circlearrowleft^{1} \cdots \circlearrowleft^{n}, *_{X}\right),\left.\quad(D, E) \longmapsto D\right|_{\circlearrowleft \rightarrow E}
$$

is well-defined follows from the STU, AS and IHX relations in the target. Besides, it is obvious that this operation is compatible with additional "link relations" on $X$. This corresponds to the operation $\diamond$ in $[S 96]$.

Corollary 5.4 (Suetsugu). Let $L \subset N$ be a null-homologous knot in a $\mathbb{Q} H S$ (with framing given by the preferred parallel), and let $P \subset S^{1} \times D^{2}$ be a framed link. Then, we have

$$
Z\left(N, L_{P}\right)=\left.\left(Z(N, L) \sharp \nu^{-1}\right)\right|_{\circlearrowleft \rightarrow Z^{\mathbf{o}_{(P)}}}
$$

where $\sharp$ denotes the usual multiplication in $\mathcal{A}(\circlearrowleft) \cong \mathcal{A}(\uparrow)$ and $\nu=Z$ (unknot).

Proof. There is a tangle $Q$ in $[-1,+1] \times D^{2}$ whose "closure" in $S^{1} \times D^{2}$ is $P$. Thus, we obtain a decomposition of the following form for $U \sqcup P \subset S^{3}$ in the strict monoidal category of tangles:

$$
U \sqcup P=\begin{array}{|l|l|}
\hline \text { "caps" } \\
\hline H & Q \\
\hline \text { "cups" } \\
\hline
\end{array}
$$

Here "caps" (resp. "cups") denote the appropriate cabling (with possible orientation changes) of the elementary tangle $\curvearrowleft$ (resp. $\curlyvee$ ), and $H$ is the "open" Hopf link whose "opened" component has been cabled accordingly:

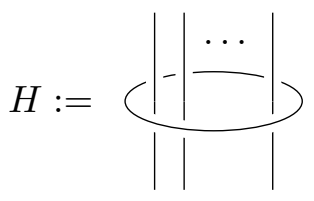

In the decomposition (5.4), $U$ is the closed component of $H$. By choosing a parenthesization of the top boundary points of $Q$, we can upgrade (5.4) to a decomposition in the non-strict monoidal category of $q$-tangles. The value of $Z(H)$ is deduced from [BL04, Theorem 4] by 
cabling the opened component of the "open" Hopf link. Then, using the definition of the invariant $Z^{\mathbf{0}}$ as it is done in [AMR98], we obtain that

$$
\chi_{u}^{-1} Z\left(S^{3}, U \sqcup P\right)=\left.\Omega_{u} \cdot \exp _{\sharp}(u)\right|_{\circlearrowleft \rightarrow Z \mathbf{o}_{(P)}} .
$$

Then, we deduce from formula ( 5.2) that

$$
\begin{aligned}
Z\left(N, L_{P}\right) & =\omega\left\langle Z^{\bowtie}(N, L) \cdot \Omega_{l}^{-1},\left.\Omega_{l} \cdot \exp _{\sharp}(-\circlearrowleft)\right|_{\circlearrowleft \rightarrow Z^{\circ} \mathbf{o}_{(P)}}\right\rangle \\
& =\omega\left\langle\partial_{\Omega_{l}}\left(Z^{ळ}(N, L) \cdot \Omega_{l}^{-1}\right),\left.\exp _{\sharp}(-\circlearrowleft)\right|_{\circlearrowleft \rightarrow Z^{\mathbf{o}_{(P)}}}\right\rangle
\end{aligned}
$$

and, by "wheeling" [BL04, Theorem 3], we get

$$
\begin{aligned}
Z\left(N, L_{P}\right) & =\omega\left\langle\chi_{l}^{-1}\left(Z(N, L) \sharp \chi_{l} \partial_{\Omega_{l}}\left(\Omega_{l}^{-1}\right)\right),\left.\exp _{\sharp}(-\circlearrowleft)\right|_{\circlearrowleft \rightarrow Z^{\circ}(P)}\right\rangle \\
& =\omega\left\langle\chi_{l}^{-1}\left(Z(N, L) \sharp\left(\chi_{l} \partial_{\Omega_{l}}\left(\Omega_{l}\right)\right)^{-1}\right),\left.\exp _{\sharp}(-\circlearrowleft)\right|_{\circlearrowleft \rightarrow Z_{(P)} \mathbf{o}_{(P)}}\right\rangle .
\end{aligned}
$$

By [BL04, Prop. 3.3], we have $\partial_{\Omega}(\Omega)=\omega \Omega$. Hence $\chi \partial_{\Omega}(\Omega)=\omega \nu$ and we conclude that

$$
Z\left(N, L_{P}\right)=\left\langle\chi_{l}^{-1}\left(Z(N, L) \sharp \nu^{-1}\right),\left.\exp _{\sharp}(-\circlearrowleft)\right|_{\circlearrowleft \rightarrow Z \mathbf{o}_{(P)}}\right\rangle=\left.\left(Z(N, L) \sharp \nu^{-1}\right)\right|_{\circlearrowleft \rightarrow Z \mathbf{o}_{(P)}} \text {. }
$$

Remark 5.5. Formula (5.3) is stated in [S96, Theorem 2.5] for the case $N:=S^{3}$. An indication of proof is given, but the factor " $\sharp \nu^{-1}$ " seems to be missing there.

Remark 5.6. If $N:=S^{3}$ and $P$ is connected in Corollary 5.4, then we can view $L$ and $P$ as one-component "bottom tangles" in handlebodies of genus 0 and 1 , respectively. In such a case, formula ( 5.3) follows immediately from the functoriality of the invariant of "bottom tangles in handlebodies" that is constructed in [HM17] as an extension of the Kontsevich integral.

\section{The CASE OF KNOTS THAT ARE NOT TRIVIAL IN HOMOLOGY}

Let now $M_{1}$ and $M_{2}$ be $\mathbb{Q H S}$ and let $K_{1} \subset M_{1}$ and $K_{2} \subset M_{2}$ be framed knots. We identify the boundary of $X_{i}=M_{i} \backslash \operatorname{Int}\left(\mathrm{N}\left(K_{i}\right)\right)$ with $\mathbb{T}$ as in (2.3) and we consider the splice

$$
M:=X_{1} \bigcup_{f} X_{2}
$$

defined by the homeomorphism $f: \mathbb{T} \rightarrow \mathbb{T}$ that is encoded by four integers $p, q, r, s$ as in (2.4). The self-linking number of $K_{i}$ is denoted by

$$
\frac{u_{i}}{v_{i}}:=\operatorname{lk}\left(K_{i}, \rho\left(K_{i}\right)\right) \in \mathbb{Q}
$$

where $u_{i}, v_{i}$ are coprime integers such that $v_{i}>0$. Observe that, when $K_{i}$ is null-homologous and $\rho\left(K_{i}\right)$ is the preferred parallel, we have $u_{i}=0$ and $v_{i}=1$.

Lemma 6.1. With the above notations, $M$ is a $\mathbb{Q} H S$ if and only if we have

$$
q u_{1} u_{2}+r v_{1} v_{2}+s u_{2} v_{1}+p v_{2} u_{1} \neq 0 .
$$

Proof. Recall that the longitude $\ell\left(K_{i}\right)$ of $K_{i}$ is the oriented simple closed curve in $\partial \mathrm{N}\left(K_{i}\right)$ that is homologous to a positive multiple of $K_{i}$ in $\mathrm{N}\left(K_{i}\right)$ and is rationally null-homologous in the exterior of $\mathrm{N}\left(K_{i}\right)$. Thus we have $\operatorname{lk}\left(K_{i}, \ell\left(K_{i}\right)\right)=0$ and we deduce from (6.2) that

$$
\ell\left(K_{i}\right)=-u_{i} \mu\left(K_{i}\right)+v_{i} \rho\left(K_{i}\right) \in H_{1}\left(\partial \mathrm{N}\left(K_{i}\right) ; \mathbb{Z}\right) .
$$


A Mayer-Vietoris argument shows that $H_{1}(M ; \mathbb{Q})$ is generated by $\mu\left(K_{2}\right)$ and $\rho\left(K_{2}\right)$, subject to the relations

$$
-u_{2} \mu\left(K_{2}\right)+v_{2} \rho\left(K_{2}\right)=0 \quad \text { and } \quad\left(-p u_{1}-r v_{1}\right) \mu\left(K_{2}\right)+\left(-q u_{1}-s v_{1}\right) \rho\left(K_{2}\right)=0
$$

which express the triviality in homology of $\ell\left(K_{2}\right)$ and $\ell\left(K_{1}\right)$, respectively. Hence $H_{1}(M ; \mathbb{Q})=0$ if and only if

$$
\left|\begin{array}{cc}
-u_{2} & v_{2} \\
p u_{1}+r v_{1} & q u_{1}+s v_{1}
\end{array}\right| \neq 0
$$

For any framed knot $L$ in a $\mathbb{Q H S} N$, with self-linking number $\varphi \in \mathbb{Q}$, we denote by

$$
\underline{Z}(N, L):=Z(N, L) \sharp \exp _{\sharp}\left(-\frac{\varphi}{2} \uparrow\right) \in \mathcal{A}\left(\circlearrowleft_{l}\right) \cong \mathcal{A}\left(\uparrow^{l}\right)
$$

the unframed version of the Kontsevich-LMO invariant $Z(N, L)$ and we denote by

$$
\underline{Z}^{\not}(N, L):=\partial_{\Omega_{l}}^{-1} \chi^{-1}(\underline{Z}(N, L)) \in \mathcal{A}\left(*_{l}\right)
$$

the wheeled version of the latter. Note that $\underline{Z}^{\not \alpha}(N, L)$ does not show any strut and, by "wheeling" [BL04, Theorem 3], we have

$$
\underline{Z}^{\not \alpha}(N, L)=Z^{\not}(N, L) \cdot \exp \left(-\frac{\varphi}{2} \bigcap_{l}+\frac{\varphi}{48} \bigcirc\right) .
$$

Theorem 6.2. For $i \in\{1,2\}$, let $K_{i} \subset M_{i}$ be a framed knot in a $\mathbb{Q} H S$, with self-linking number $u_{i} / v_{i} \in \mathbb{Q}$, and let $M$ be the splice of $\left(M_{1}, K_{1}\right)$ and $\left(M_{2}, K_{2}\right)$ as described by (6.1) in terms of four integers $p, q, r, s$. If $M$ is also a $\mathbb{Q} H S$, then we have

$$
Z(M)=\omega \exp \left(\frac{\theta \kappa}{48}\right)\left\langle\left.\partial_{D_{1}}\left(\underline{Z}^{\not}\left(K_{1}\right)\right)\right|_{k_{1} \rightarrow-v_{1} v_{2} k_{2} / \lambda}, \partial_{D_{2}}\left(\underline{Z}^{\not}\left(K_{2}\right)\right)\right\rangle,
$$

where we have set $\lambda:=q u_{1} u_{2}+r v_{1} v_{2}+s u_{2} v_{1}+p v_{2} u_{1}, \tau_{1}:=q u_{1}+s v_{1}, \tau_{2}:=q u_{2}+p v_{2}$,

$$
\kappa:= \begin{cases}S(s / q)-(s+p) / q+3 \operatorname{sgn}\left(q \tau_{1}\right)+3 \operatorname{sgn}\left(\lambda \tau_{1}\right)-u_{1} / v_{1}-u_{2} / v_{2} & \text { if } q \neq 0, \\ s(3 \operatorname{sgn}(\lambda)-r)-u_{1} / v_{1}-u_{2} / v_{2} & \text { if } q=0,\end{cases}
$$

and

$$
D_{1}:=\exp \left(-\frac{v_{1} \tau_{2}}{2 \lambda} \cup^{k_{1} k_{1}}\right), \quad D_{2}:=\exp \left(-\frac{v_{2} \tau_{1}}{2 \lambda} \cup^{k_{2}}\right)
$$

Proof. The first part of the proof of Theorem 3.1 works verbatim in the more general situation that we are now considering. Thus we choose a matrix decomposition as in (2.5), which leads to $n \geq 1$ integers $a_{1}, \ldots, a_{n}$, and we consider the Hopf chain $H:=H\left(a_{1}, \ldots, a_{n}\right) \subset M_{1} \sharp M_{2}$ that "clasps" positively $K_{1}$ and $\epsilon_{n} K_{2}$. We obtain that

$$
\begin{aligned}
& Z(M)=\omega \exp \left(\frac{\theta\left(3 \zeta(\Lambda)-\sum_{i=1}^{n} a_{i}\right)}{48}\right) \int Z^{ळ}\left(K_{1}\right) Z^{ळ}\left(\epsilon_{n} K_{2}\right) \cdot \\
& \cdot \exp \left(\underset{k_{1} h_{1}}{\cap}+\sum_{i=1}^{n-1} \underset{h_{i} h_{i+1}}{\cap}+\underset{h_{n} k_{2}}{\cap}+\frac{1}{2} \sum_{i=1}^{n} a_{i} \underset{h_{i} h_{i}}{\cap}\right) \mathrm{d} k_{1} \mathrm{~d} h_{1} \cdots \mathrm{d} h_{n} \mathrm{~d} k_{2} .
\end{aligned}
$$


Here $\Lambda$ denotes the linking matrix of $K_{1} \sqcup H \sqcup \epsilon_{n} K_{2}$ in $M_{1} \sharp M_{2}$, which is the tridiagonal matrix

$$
\Lambda:=\left(\begin{array}{cccccc}
u_{1} / v_{1} & 1 & 0 & \cdots & 0 & 0 \\
1 & a_{1} & 1 & \cdots & 0 & 0 \\
0 & 1 & a_{2} & \cdots & 0 & 0 \\
\vdots & \vdots & \vdots & \ddots & \vdots & \vdots \\
0 & 0 & 0 & \cdots & a_{n} & 1 \\
0 & 0 & 0 & \cdots & 1 & u_{2} / v_{2}
\end{array}\right) .
$$

Using (6.3), we deduce that

$$
\begin{aligned}
& Z(M)=\omega \exp \left(\frac{\theta(3 \zeta(\Lambda)-\operatorname{tr}(\Lambda))}{48}\right) \int \underline{Z}^{\not 2}\left(K_{1}\right) \underline{Z}^{\not}\left(\epsilon_{n} K_{2}\right) \cdot \\
& \cdot \exp \left(\underset{k_{1} h_{1}}{\cap}+\sum_{i=1}^{n-1} \underset{h_{i} h_{i+1}}{\cap}+\underset{h_{n} k_{2}}{\cap}+\frac{1}{2} \sum_{i=1}^{n} a_{i} \underset{h_{i} h_{i}}{\cap}+\frac{u_{1}}{2 v_{1}} \underset{k_{1} k_{1}}{\cap}+\frac{u_{2}}{2 v_{2}} \underset{k_{2} k_{2}}{\cap}\right) \text {. } \\
& \cdot \mathrm{d} k_{1} \mathrm{~d} h_{1} \cdots \mathrm{d} h_{n} \mathrm{~d} k_{2}
\end{aligned}
$$

The $2 \times 2$ matrix associated to the tridiagonal matrix $\Lambda$ is

$$
\begin{aligned}
& \left(\begin{array}{cc}
0 & -1 \\
1 & 0
\end{array}\right) \cdot\left(\begin{array}{cc}
u_{1} / v_{1} & -1 \\
1 & 0
\end{array}\right)\left(\begin{array}{cc}
a_{1} & -1 \\
1 & 0
\end{array}\right) \cdots\left(\begin{array}{cc}
a_{n} & -1 \\
1 & 0
\end{array}\right)\left(\begin{array}{cc}
u_{2} / v_{2} & -1 \\
1 & 0
\end{array}\right) \\
& =\left(\begin{array}{cc}
0 & -1 \\
1 & 0
\end{array}\right)\left(\begin{array}{cc}
u_{1} / v_{1} & -1 \\
1 & 0
\end{array}\right)\left(\begin{array}{cc}
1 & 0 \\
0 & -1
\end{array}\right)\left(\begin{array}{cc}
a_{1} & 1 \\
-1 & 0
\end{array}\right) \cdots\left(\begin{array}{cc}
a_{n} & 1 \\
-1 & 0
\end{array}\right)\left(\begin{array}{cc}
1 & 0 \\
0 & -1
\end{array}\right)\left(\begin{array}{cc}
u_{2} / v_{2} & -1 \\
1 & 0
\end{array}\right) \\
& =\left(\begin{array}{cc}
0 & -1 \\
1 & 0
\end{array}\right)\left(\begin{array}{cc}
u_{1} / v_{1} & 1 \\
1 & 0
\end{array}\right)\left(\left(\begin{array}{cc}
a_{n} & -1 \\
1 & 0
\end{array}\right) \cdots\left(\begin{array}{cc}
a_{1} & -1 \\
1 & 0
\end{array}\right)\right)^{T}\left(\begin{array}{cc}
u_{2} / v_{2} & -1 \\
-1 & 0
\end{array}\right) \\
& \stackrel{(2.5)}{=}\left(\begin{array}{cc}
0 & -1 \\
1 & 0
\end{array}\right)\left(\begin{array}{cc}
u_{1} / v_{1} & 1 \\
1 & 0
\end{array}\right)\left(\begin{array}{cc}
q & s \\
-p & -r
\end{array}\right)^{T}\left(\begin{array}{cc}
u_{2} / v_{2} & -1 \\
-1 & 0
\end{array}\right) \\
& =\left(\begin{array}{cc}
-\left(q u_{2}+p v_{2}\right) / v_{2} & q \\
\left(q u_{1} u_{2}+s u_{2} v_{1}+p u_{1} v_{2}+r v_{1} v_{2}\right) /\left(v_{1} v_{2}\right) & -\left(q u_{1}+s v_{1}\right) / v_{1}
\end{array}\right)
\end{aligned}
$$

so that, by Proposition A.1, we have

$$
\Lambda^{-1}=\frac{1}{\lambda}\left(\begin{array}{ccccc}
v_{1} \tau_{2} & ? & \cdots & ? & (-1)^{n+1} v_{1} v_{2} \\
? & ? & \cdots & ? & ? \\
\vdots & \vdots & \ddots & \vdots & \vdots \\
? & ? & \cdots & ? & ? \\
(-1)^{n+1} v_{1} v_{2} & ? & \cdots & ? & v_{2} \tau_{1}
\end{array}\right)
$$

where $\lambda:=q u_{1} u_{2}+s u_{2} v_{1}+p u_{1} v_{2}+r v_{1} v_{2}, \tau_{1}:=q u_{1}+s v_{1}$ and $\tau_{2}:=q u_{2}+p v_{2}$. Therefore, performing Gaussian integration and using that $\underline{Z}^{\not \infty}\left(-K_{2}\right)$ is obtained from $\underline{Z}^{\infty}\left(K_{2}\right)$ by the change $k_{2} \rightarrow-k_{2}$, we get

$$
\begin{aligned}
Z(M)= & \omega \exp \left(\frac{\theta(3 \zeta(\Lambda)-\operatorname{tr}(\Lambda))}{48}\right) \cdot \\
& \cdot\left\langle\exp \left(-\frac{v_{1} \tau_{2}}{2 \lambda} \cup^{k_{1}} \cup^{k_{1}}-\frac{v_{1} v_{2}}{\lambda} \cup^{k_{1} k_{2}}-\frac{v_{2} \tau_{1}}{2 \lambda} \cup^{k_{2}}\right), \underline{Z}^{k_{2}}\left(K_{1}\right) \underline{Z}^{\not}\left(K_{2}\right)\right\rangle .
\end{aligned}
$$


Using the notation (6.4), this is equivalent to

$$
Z(M)=\omega \exp \left(\frac{\theta(3 \zeta(\Lambda)-\operatorname{tr}(\Lambda))}{48}\right) \cdot\left\langle\left.\partial_{D_{1}}\left(\underline{Z}^{\rightsquigarrow}\left(K_{1}\right)\right)\right|_{k_{1} \rightarrow-v_{1} v_{2} k_{2} / \lambda}, \partial_{D_{2}}\left(\underline{Z}^{\not}\left(K_{2}\right)\right)\right\rangle .
$$

It remains to compute $\kappa:=3 \zeta(\Lambda)-\operatorname{tr}(\Lambda) \in \mathbb{Q}$. By the above computation of the $2 \times 2$ matrix $A\left(u_{1} / v_{1}, a_{1}, \ldots, a_{n}, u_{2} / v_{2}\right)$ associated to $\Lambda=\Lambda\left(u_{1} / v_{1}, a_{1}, \ldots, a_{n}, u_{2} / v_{2}\right)$, we deduce from (A.1) that

$$
\zeta(\Lambda)=\zeta\left(\Lambda\left(u_{1} / v_{1}, a_{1}, \ldots, a_{n}\right)\right)+\operatorname{sgn}\left(\lambda \tau_{1}\right) .
$$

Besides, since the $2 \times 2$ matrix associated to $\Lambda\left(u_{1} / v_{1}, a_{1}, \ldots, a_{n}\right)$ is

$$
\begin{aligned}
& A\left(u_{1} / v_{1}, a_{1}, \ldots, a_{n}, u_{2} / v_{2}\right)\left(\begin{array}{cc}
0 & 1 \\
-1 & u_{2} / v_{2}
\end{array}\right) \\
= & \left(\begin{array}{cc}
-\tau_{2} / v_{2} & q \\
\lambda /\left(v_{1} v_{2}\right) & -\tau_{1} / v_{1}
\end{array}\right)\left(\begin{array}{cc}
0 & 1 \\
-1 & u_{2} / v_{2}
\end{array}\right)=\left(\begin{array}{cc}
-q & -p \\
\tau_{1} / v_{1} & \left(p u_{1}+r v_{1}\right) / v_{1}
\end{array}\right),
\end{aligned}
$$

it follows from ( A.2) that

$$
\zeta\left(\Lambda\left(u_{1} / v_{1}, a_{1}, \ldots, a_{n}\right)\right)=\zeta\left(\Lambda\left(a_{1}, \ldots, a_{n}\right)\right)+\operatorname{sgn}\left(q \tau_{1}\right) .
$$

Finally, since the $2 \times 2$ matrix associated to $\Lambda\left(a_{1}, \ldots, a_{n}\right)$ is

$$
\begin{aligned}
& \left(\begin{array}{cc}
0 & -1 \\
1 & 0
\end{array}\right) \cdot\left(\begin{array}{cc}
a_{1} & -1 \\
1 & 0
\end{array}\right) \cdots\left(\begin{array}{cc}
a_{n} & -1 \\
1 & 0
\end{array}\right) \\
= & \left(\begin{array}{cc}
0 & -1 \\
1 & 0
\end{array}\right)\left(\begin{array}{cc}
1 & 0 \\
0 & -1
\end{array}\right)\left(\begin{array}{cc}
a_{1} & 1 \\
-1 & 0
\end{array}\right) \cdots\left(\begin{array}{cc}
a_{n} & 1 \\
-1 & 0
\end{array}\right)\left(\begin{array}{cc}
1 & 0 \\
0 & -1
\end{array}\right) \\
\stackrel{(2.5)}{=} & \left(\begin{array}{ll}
0 & 1 \\
1 & 0
\end{array}\right)\left(\begin{array}{cc}
q & s \\
-p & -r
\end{array}\right)^{T}\left(\begin{array}{cc}
1 & 0 \\
0 & -1
\end{array}\right)=\left(\begin{array}{ll}
s & r \\
q & p
\end{array}\right),
\end{aligned}
$$

we deduce from Theorem A.3 that

$$
3 \zeta\left(\Lambda\left(a_{1}, \ldots, a_{n}\right)\right)-\sum_{i=1}^{n} a_{i}= \begin{cases}S(s / q)-(s+p) / q & \text { if } q \neq 0 \\ -r / s & \text { if } q=0\end{cases}
$$

We conclude that

$$
\kappa= \begin{cases}S(s / q)-(s+p) / q+3 \operatorname{sgn}\left(q \tau_{1}\right)+3 \operatorname{sgn}\left(\lambda \tau_{1}\right)-u_{1} / v_{1}-u_{2} / v_{2} & \text { if } q \neq 0 \\ -r / s+3 \operatorname{sgn}(\lambda s)-u_{1} / v_{1}-u_{2} / v_{2} & \text { if } q=0\end{cases}
$$

Remark 6.3. At the end of the proof of Theorem 6.2, we have applied (A.1) before (A.2) to compute $\kappa \in \mathbb{Q}$. If we went the other way, we would have obtained an equivalent formula:

$$
\kappa= \begin{cases}S(s / q)-(s+p) / q+3 \operatorname{sgn}\left(q \tau_{2}\right)+3 \operatorname{sgn}\left(\lambda \tau_{2}\right)-u_{1} / v_{1}-u_{2} / v_{2} & \text { if } q \neq 0 \\ p(3 \operatorname{sgn}(\lambda)-r)-u_{1} / v_{1}-u_{2} / v_{2} & \text { if } q=0 .\end{cases}
$$

Remark 6.4. In the same way as Theorem 3.1 extends to Theorem 5.1, there is a generalization of Theorem 6.2 which includes additional links $L_{i}$ in $M_{i}$ (disjoint from $K_{i}$ ). 


\section{Appendix A. Signatures of tridiagonal matrices}

A tridiagonal matrix is a square matrix of the form

$$
\Lambda\left(c_{1}, \ldots, c_{\ell}\right):=\left(\begin{array}{cccccc}
c_{1} & 1 & 0 & \ldots & \ldots & 0 \\
1 & c_{2} & 1 & \ddots & & \vdots \\
0 & 1 & c_{3} & 1 & \ddots & \vdots \\
\vdots & \ddots & \ddots & \ddots & \ddots & 0 \\
\vdots & & \ddots & \ddots & \ddots & 1 \\
0 & \ldots & \ldots & 0 & 1 & c_{\ell}
\end{array}\right)
$$

where $c_{1}, \ldots, c_{\ell} \in \mathbb{R}$. The associated $2 \times 2$ matrix is

$$
\mathrm{A}\left(c_{1}, \ldots, c_{\ell}\right):=\left(\begin{array}{cc}
0 & -1 \\
1 & 0
\end{array}\right) \cdot\left(\begin{array}{cc}
c_{1} & -1 \\
1 & 0
\end{array}\right)\left(\begin{array}{cc}
c_{2} & -1 \\
1 & 0
\end{array}\right) \cdots\left(\begin{array}{cc}
c_{\ell} & -1 \\
1 & 0
\end{array}\right) \in \mathrm{SL}_{2}(\mathbb{R}) .
$$

Proposition A.1 (Bar-Natan \& Lawrence). Let $c_{1}, \ldots, c_{\ell} \in \mathbb{R}$ and write

$$
\left(\begin{array}{ll}
\alpha & \beta \\
\gamma & \delta
\end{array}\right):=\mathrm{A}\left(c_{1}, \ldots, c_{\ell}\right)
$$

The matrix $\Lambda\left(c_{1}, \ldots, c_{\ell}\right)$ is invertible if and only if $\gamma \neq 0$. In such a case, the four "corners" of the inverse of $\Lambda\left(c_{1}, \ldots, c_{\ell}\right)$ are given by

$$
\Lambda\left(c_{1}, \ldots, c_{\ell}\right)^{-1}=\left(\begin{array}{ccccc}
-\alpha / \gamma & ? & \cdots & ? & (-1)^{\ell+1} / \gamma \\
? & ? & \cdots & ? & ? \\
\vdots & \vdots & \ddots & \vdots & \vdots \\
? & ? & \cdots & ? & ? \\
(-1)^{\ell+1} / \gamma & ? & \cdots & ? & -\delta / \gamma
\end{array}\right)
$$

Proof. The arguments given in [BL04, Prop. 2.4] under the assumption $c_{1}, \ldots, c_{\ell} \in \mathbb{Z}$ work in the same way for arbitrary $c_{1}, \ldots, c_{\ell} \in \mathbb{R}$. But it seems that the $(-1)^{\ell}$ in the statement of [BL04, Prop. 2.4] should be replaced by $(-1)^{\ell+1}$.

We explain how to compute inductively the signature $\zeta(\Lambda)$ of a tridiagonal matrix $\Lambda$.

Proposition A.2. Let $c_{1}, \ldots, c_{\ell} \in \mathbb{R}$ and write

$$
\left(\begin{array}{ll}
\alpha & \beta \\
\gamma & \delta
\end{array}\right):=\mathrm{A}\left(c_{1}, \ldots, c_{\ell}\right)
$$

Then we have the following inductive formulas:

$$
\begin{aligned}
\zeta\left(\Lambda\left(c_{1}, c_{2}, \ldots, c_{\ell}\right)\right) & =\zeta\left(\Lambda\left(c_{1}, \ldots, c_{\ell-1}\right)\right)-\operatorname{sgn}(\gamma) \operatorname{sgn}(\delta) \\
\zeta\left(\Lambda\left(c_{1}, c_{2}, \ldots, c_{\ell}\right)\right) & =\zeta\left(\Lambda\left(c_{2}, \ldots, c_{\ell}\right)\right)-\operatorname{sgn}(\gamma) \operatorname{sgn}(\alpha) .
\end{aligned}
$$

Proof. The proof of (A.1) being very similar to the proof of (A.2), we give the latter and omit the former. A straightforward computation gives

$$
A\left(c_{2}, \ldots, c_{\ell}\right)=\left(\begin{array}{cc}
\gamma+\alpha c_{1} & \delta+\beta c_{1} \\
-\alpha & -\beta
\end{array}\right) .
$$

Thus, by Proposition A.1, the matrix $\underline{\Lambda}:=\Lambda\left(c_{2}, \ldots, c_{\ell}\right)$ is invertible if and only if $\alpha \neq 0$. 
Assume first that $\alpha \neq 0$. We shall use the fact that $\Lambda:=\Lambda\left(c_{1}, c_{2}, \ldots, c_{\ell}\right)$ is obtained from $\underline{\Lambda}$ by a kind of "plumbing" operation - compare with the proof of [GR16, Prop. 2.5.3]. Specifically, we have the following congruence where $v:=(1,0, \ldots, 0) \in \mathbb{R}^{\ell-1}$ :

$$
\underbrace{\left(\begin{array}{cc}
c_{1} & v \\
v^{T} & \underline{\Lambda}
\end{array}\right)}_{\Lambda}=\left(\begin{array}{cc}
1 & v \underline{\Lambda}^{-1} \\
0 & I
\end{array}\right)\left(\begin{array}{cc}
c_{1}-v \underline{\Lambda}^{-1} v^{T} & 0 \\
0 & \underline{\Lambda}
\end{array}\right)\left(\begin{array}{cc}
1 & 0 \\
\underline{\Lambda}^{-1} v^{T} & I
\end{array}\right) .
$$

Therefore

$$
\zeta(\Lambda)=\operatorname{sgn}\left(c_{1}-v \underline{\Lambda}^{-1} v^{T}\right)+\zeta(\underline{\Lambda}) .
$$

By Proposition A.1, the upper left "corner" of $\underline{\Lambda}^{-1}$ can be read from (A.3). Hence we get $v \underline{\Lambda}^{-1} v^{T}=\gamma / \alpha+c_{1}$ and we deduce that $\zeta(\Lambda)=-\operatorname{sgn}(\gamma / \alpha)+\zeta(\underline{\Lambda})$.

Assume now that $\alpha=0$. Let $x=\left(x_{2}, \ldots, x_{\ell}\right) \in \mathbb{R}^{\ell-1}$ be such that $x \neq 0$ and $\underline{\Lambda} x^{T}=0$. By writing the linear system $\underline{\Lambda} x^{T}=0$, we see that $x_{2}=0$ would successively imply $x_{3}=0$, $\ldots, x_{\ell}=0$. Hence we must have $x_{2} \neq 0$ and we get a new basis of $\mathbb{R}^{\ell}$ by substituting $x \in\{0\} \oplus \mathbb{R}^{\ell-1} \subset \mathbb{R}^{\ell}$ to the second vector of the canonical basis: we shall express in this new basis the symmetric bilinear form in $\mathbb{R}^{\ell}$ that is given by $\Lambda$ in the canonical basis. Specifically, we have the following congruence where $\underline{x}:=\left(x_{3}, \ldots, x_{\ell}\right), w:=(1,0, \ldots, 0) \in \mathbb{R}^{\ell-2}$ and $\underline{\underline{\Lambda}}:=\Lambda\left(c_{3}, \ldots, c_{\ell}\right)$ :

$$
\left(\begin{array}{ll}
0 & 0 \\
0 & \underline{\Lambda}
\end{array}\right)=\underbrace{\left(\begin{array}{cc}
x_{2} & \underline{x} \\
0 & I
\end{array}\right)}_{X^{T}} \underbrace{\left(\begin{array}{cc}
c_{2} & w \\
w^{T} & \underline{\Lambda}
\end{array}\right)}_{\underline{\Lambda}} \underbrace{\left(\begin{array}{cc}
x_{2} & 0 \\
\underline{x}^{T} & I
\end{array}\right)}_{X}
$$

Therefore, we also have the following congruence:

$$
\left(\begin{array}{ccc}
c_{1} & x_{2} & 0 \\
x_{2} & 0 & 0 \\
0 & 0 & \underline{\Lambda}
\end{array}\right)=\left(\begin{array}{cc}
1 & 0 \\
0 & X^{T}
\end{array}\right) \underbrace{\left(\begin{array}{cc}
c_{1} & v \\
v^{T} & \underline{\Lambda}
\end{array}\right)}_{\Lambda}\left(\begin{array}{cc}
1 & 0 \\
0 & X
\end{array}\right)
$$

We deduce from ( A.4) that $\zeta(\underline{\underline{\Lambda}})=\zeta(\underline{\Lambda})$ and we deduce from ( A.5) that $\zeta(\underline{\underline{\Lambda}})=\zeta(\Lambda)$.

We now recall how signatures of tridiagonal matrices with integral coefficients can be computed from Dedekind sums. For any pair of coprime integers $p, q$ with $q \neq 0$, define the Dedekind sum $\mathfrak{s}(p, q)$ by

$$
\mathfrak{s}(p, q):=\sum_{k=1}^{|q|-1}\left(\left(\frac{k}{q}\right)\right) \cdot\left(\left(\frac{k p}{q}\right)\right)
$$

where $((-))$ denotes the sawtooth function defined by $((x)):=x-\lfloor x\rfloor-1 / 2$ for $x \in \mathbb{R} \backslash \mathbb{Z}$ and $((x)):=0$ for $x \in \mathbb{Z}$. Then the Dedekind symbol

$$
S(p / q):=12 \operatorname{sgn}(q) \mathfrak{s}(p, q)
$$

is easily seen to satisfy $S(-p / q)=-S(p / q)$ and $S(p / q+1)=S(p / q)$. Furthermore, we have

$$
S(p / q)+S(q / p)=p / q+q / p+1 / p q-3 \operatorname{sgn}(p q) ;
$$

see [KM94] and references therein.

The following theorem is proved in [KM94, (1.12) \& (2.2)] — see also [BG92]. For the sake of completeness, we provide a proof based on Proposition A.2 and assuming the reciprocity law ( A.6). 
Theorem A.3 (Kirby \& Melvin). Let $c_{1}, \ldots, c_{\ell} \in \mathbb{Z}$ and write

$$
\left(\begin{array}{ll}
\alpha & \beta \\
\gamma & \delta
\end{array}\right):=\mathrm{A}\left(c_{1}, \ldots, c_{\ell}\right)
$$

The signature $\zeta(\Lambda)$ and the trace $\operatorname{tr}(\Lambda)$ of $\Lambda:=\Lambda\left(c_{1}, \ldots, c_{\ell}\right)$ are related as follows:

$$
3 \zeta(\Lambda)-\operatorname{tr}(\Lambda)= \begin{cases}S(\alpha / \gamma)-(\alpha+\delta) / \gamma & \text { if } \gamma \neq 0 \\ -\beta / \alpha & \text { if } \gamma=0\end{cases}
$$

Proof. The proof is by induction on $\ell \geq 1$. For $\ell=1$, ( A.7) asserts that

$$
3 \operatorname{sgn}\left(c_{1}\right)-c_{1}= \begin{cases}-S\left(1 / c_{1}\right)+2 / c_{1} & \text { if } c_{1} \neq 0 \\ 0 & \text { if } c_{1}=0\end{cases}
$$

which is trivial for $c_{1}=0$ and follows from ( A.6) for $c_{1} \neq 0$. Hence we suppose that ( A.7) holds true for $\underline{\Lambda}:=\Lambda\left(c_{2}, \ldots, c_{\ell}\right)$; the $2 \times 2$ matrix associated to $\underline{\Lambda}$ has been computed at (A.3).

Assume that $\gamma=0$. It follows from (A.2) that

$$
3 \zeta(\Lambda)-\operatorname{tr}(\Lambda)=3 \zeta(\underline{\Lambda})-\operatorname{tr}(\underline{\Lambda})-c_{1} .
$$

Since $\alpha \delta-\beta \gamma=1$, we must have $\alpha \neq 0$. Then the induction hypothesis gives

$$
3 \zeta(\Lambda)-\operatorname{tr}(\Lambda)=S\left(-\gamma / \alpha-c_{1}\right)-\left(\gamma+\alpha c_{1}-\beta\right) /(-\alpha)-c_{1}=-\beta / \alpha .
$$

Assume now that $\gamma \neq 0$. If $\alpha=0$, we have $\beta=-\gamma \in\{-1,+1\}$ and it follows from ( A.2) and the induction hypothesis that

$$
\begin{aligned}
3 \zeta(\Lambda)-\operatorname{tr}(\Lambda) & =3 \zeta(\underline{\Lambda})-\operatorname{tr}(\underline{\Lambda})-c_{1} \\
& =-\left(\delta+\beta c_{1}\right) /\left(\gamma+\alpha c_{1}\right)-c_{1}=-\delta / \gamma=S(\alpha / \gamma)-(\alpha+\delta) / \gamma .
\end{aligned}
$$

If $\alpha \neq 0$, it follows from (A.2) and the induction hypothesis that

$$
\begin{aligned}
3 \zeta(\Lambda)-\operatorname{tr}(\Lambda) & =3 \zeta(\underline{\Lambda})-\operatorname{tr}(\underline{\Lambda})-3 \operatorname{sgn}(\gamma \alpha)-c_{1} \\
& =S\left(\left(\gamma+c_{1} \alpha\right) /(-\alpha)\right)-\left(\gamma+c_{1} \alpha-\beta\right) /(-\alpha)-3 \operatorname{sgn}(\gamma \alpha)-c_{1} \\
& =-S(\gamma / \alpha)+\gamma / \alpha-\beta / \alpha-3 \operatorname{sgn}(\gamma \alpha) \\
& \stackrel{\text { A. } 6)}{=} S(\alpha / \gamma)-\alpha / \gamma-1 /(\gamma \alpha)-\beta / \alpha=S(\alpha / \gamma)-\alpha / \gamma-\delta / \gamma .
\end{aligned}
$$

\section{REFERENCES}

[AMr98] J. Andersen, J. Mattes \& N. Reshetikhin - "Quantization of the algebra of chord diagrams", Math. Proc. Cambridge Philos. Soc. 124 (1998), no. 3, p. 451-467.

[BGRT02] D. Bar-Natan, S. Garoufalidis, L. Rozansky \& D. Thurston - "The Aarhus integral of rational homology 3-spheres. II. Invariance and universality", Selecta Math. (N.S.) 8 (2002), no. 3, p. 341371.

[BGRT04] D. Bar-Natan, S. Garoufalidis, L. Rozansky \& D. Thurston - "The Aarhus integral of rational homology 3-spheres. III. Relation with the Le-Murakami-Ohtsuki invariant.", Selecta Math. (N.S.) 10 (2004), no. 3, p. 305-324.

[BL04] D. BAR-NATAN \& R. LAWREnCE - "A rational surgery formula for the LMO invariant", Israel J. Math. 140 (2004), p. 29-60.

[BLT03] D. Bar-Natan, T. Le \& D. Thurston - "Two applications of elementary knot theory to Lie algebras and Vassiliev invariants", Geom. Topol. 7 (2003), p. 1-31.

[BG92] J. Barge \& E. Ghys - "Cocycles d'Euler et de Maslov", Math. Ann. 294 (1992), no. 2, p. $235-265$.

[BH09] H. Boden \& B. Himpel - "Splitting the spectral flow and the SU(3) Casson invariant for spliced sums", Alg. Geom. Topol. 9 (2009), no. 2, p. 865-902.

[BN90] S. BOYER \& A. NiCAS - "Varieties of group representations and Casson's invariant for rational homology 3-spheres", Trans. Amer. Math. Soc. 322 (1990), no. 2, p. 507-522. 
[CG88] T. Cochran \& R. GompF - "Applications of Donaldson's theorems to classical knot concordance, homology 3-spheres and property P", Topology 27 (1988), no. 4, p. 495-512.

[EN85] D. Eisenbud \& W. Neumann - "Three-dimensional link theory and invariants of plane curve singularities", Annals of Mathematics Studies 110. Princeton University Press, Princeton, NJ, 1985.

[F93] G. FuJitA - "A splicing formula for Casson-Walker's invariant", Math. Ann. 296 (1993), no. 2, p. 327338.

[FN88] S. Fukuhara \& N. Maruyama - "A sum formula for Casson's $\lambda$-invariant", Tokyo J. Math. 11 (1988), no. 2, p. 281-287.

[GGP01] S. Garoufalidis, M. Goussarov \& M. Polyak - "Calculus of clovers and finite type invariants of 3-manifolds", Geom. Topol. 5 (2001), p. 75-108.

[GR16] E. Ghys \& A. RANicki - "Signatures in algebra, topology and dynamics", Six papers on signatures, braids and Seifert surfaces, Ensaios Mat., 30, Soc. Brasil. Mat., Rio de Janeiro, p. 1-173 (2016).

[G75] C. Gordon - "Knots, homology spheres, and contractible 4-manifolds", Topology 14 (1975), p. 151-172.

[H00] K. Habiro - "Claspers and finite type invariants of links", Geom. Topol. 4 (2000), p. 1-83.

[HM17] K. Habiro \& G. Massuyeau - "The Kontsevich integral for bottom tangles in handlebodies", preprint (2017), arXiv:1702.00830v4.

[I17] T. Iто - "On LMO invariant constraints for cosmetic surgery and other surgery problems for knots in $S^{3 ",}$, preprint (2017), arXiv:1704.01755v2.

[KM94] R. Kirby \& P. Melvin - "Dedekind sums, $\mu$-invariants and the signature cocycle", Math. Ann. 299 (1994), no. 2, p. 231-267.

[K00] A. KRICKER - "The lines of the Kontsevich integral and Rozansky's rationality conjecture", preprint (2000), arXiv:math/0005284v1.

[Le97] T. LE - "An invariant of integral homology 3-spheres which is universal for all finite type invariants", Solitons, geometry, and topology: on the crossroad, Amer. Math. Soc. Transl. Ser. 2 179, Amer. Math. Soc., Providence, RI, p. 75-100 (1997).

[LMO98] T. Le, J. Murakami \& T. Ohtsuki - "On a universal perturbative invariant of 3-manifolds, Topology 37 (1998), no. 3, p. 539-574.

[M14] G. Massuyeau - "Splitting formulas for the LMO invariant of rational homology three-spheres", Alg. Geom. Topol. 14 (2014), no. 4, p. 3553-3588.

[M12a] D. Moussard - "On Alexander modules and Blanchfield forms of null-homologous knots in rational homology spheres", J. Knot Th. Ramifications 21 (2012), no. 5, 1250042, 21 pp.

[M12b] D. Moussard - "Finite type invariants of rational homology 3-spheres", Alg. Geom. Topol. 12 (2012), no. 4, p. 2389-2428.

[O96a] T. Oнtsuki - "Finite type invariants of integral homology 3-spheres", J. Knot Th. Ramifications 5 (1996), no. 1, p. 101-115.

[O96b] T. Oнtsuki - "A polynomial invariant of rational homology 3-spheres", Invent. Math. 123 (1996), no. 2, p. 241-257.

[S96] Y. Suetsugu - "Kontsevich invariant for links in a donut and links of satellite form", Osaka J. Math. 33 (1996), no. 4, p. 823-828.

[W92] K. WAlker - "An extension of Casson's invariant", Annals of Mathematics Studies 126, Princeton University Press, Princeton, NJ, 1992.

Institut de Mathématiques de Bourgogne, UMR 5584, CNRS, Université Bourgogne FrancheComté, 21000 Dijon, France

E-mail address: gwenael.massuyeau@u-bourgogne.fr

Institut de Mathématiques de Marseille, UMR 7373, Université D'Aix-Marseille, Marseille, FRANCE

E-mail address: delphine.moussard@univ-amu.fr 